\title{
THE GAUSSIAN LAW AND THE LAW OF THE ITERATED LOGARITHM FOR LACUNARY SETS OF CHARACTERS
}

\author{
BY
}

\author{
E. DUDLEY
}

\begin{abstract}
Salem and Zygmund showed that the Gaussian law holds for Hadamard sequences of real numbers while Mary Weiss proved a similar result for the law of the iterated logarithm. In the present paper, the author obtains corresponding results for lacunary sets of characters of an arbitrary infinite compact abelian group. It is shown that the laws are best satisfied for a certain class of lacunary sets but that modified results apply to more general classes.
\end{abstract}

1.1. Edwards, Hewitt and Ross [1] conjecture that general classes of lacunary sets of characters of a compact abelian group exhibit properties similar to those known to hold for Hadamard sets of integers. Such a property which has been shown to hold in the abstract setting is the Fatou-Zygmund property (see Edwards, Hewitt and Ross [2] and Drury [1]). Two theorems describing the behaviour of Hadamard sequences are analogous to well-known theorems in probability theory dealing with sequences of independent random variables. These are the Gaussian law or Liapounov's theorem (see Gnedenko [1, §42]) and the law of the iterated logarithm or Kolmogorov's theorem (see Kolmogorov [1]). In this paper we demonstrate that both theorems hold for certain subclasses of Steckin sets of characters of an arbitrary infinite abelian group. R. C. Baker [1] has produced results in connection with the Gaussian law. We clarify and extend these results. We begin by stating the theorems we will be concerned with as they apply to sequences of independent random variables on a probability space.

1.2. Liapounov's Theorem (The GaUSSian law). Let $P$ be a probability measure on a probability space $\Omega$ and let $\left\{f_{k}\right\}_{k=1}^{\infty}$ be a sequence of bounded

Received by the editors August 29, 1974.

AMS (MOS) subject classifications (1970). Primary 42A44, 60F15, 60F05; Secondary 43A40, $42 \mathrm{~A} 36$.

Key words and phrases. Compact abelian group, lacunary sets of characters, Steckin sets, dissociate sets, Hadamard sequences, normal distribution, integral estimates, dyadic representation of natural numbers. 
independent random variables such that $\int_{\Omega} f_{k} d P=0$ for each $k=1,2, \ldots$ We set $S_{n}=\sum_{k=1}^{n} f_{k}, B_{n}=\left(\sum_{k=1}^{n} \int_{\Omega} f_{k}^{2} d P\right)^{1 / 2}$ and $M_{n}=\max _{k=1,2, \ldots, n}\left\|f_{k}\right\|_{\infty}$. Then if it is true that (i) $B_{n} \rightarrow \infty$ as $n \rightarrow \infty$, and (ii) $M_{n} B_{n}^{-1}=o$ (1) as $n \rightarrow \infty$, it follows that

$$
\lim _{n \rightarrow \infty} P\left\{t: S_{n}(t)<y B_{n}\right\}=(2 \pi)^{-1 / 2} \int_{-\infty}^{y} \exp \left(-t^{2} / 2\right) d t
$$

for all real numbers $y$.

The function on the right side of the above equality is called the normal distribution with variance 1 and will be denoted by the symbol $G_{1}(y)$. The theorem stated is less general than that appearing in Gnedenko but is the most appropriate formulation for our purposes.

1.3. KOLMOGOROV'S THEOREM (THE LAW OF THE ITERATED LOGARITHM). Let $\Omega, P,\left\{f_{k}\right\}_{k=1}^{\infty}, S_{n}, B_{n}$ and $M_{n}$ be as in 1.2. Then if it is true that (i) $B_{n}$ $\rightarrow \infty$, and (ii) $M_{n} B_{n}^{-1}\left(\log \log B_{n}\right)^{1 / 2}=o$ (1) as $n \rightarrow \infty$, we have

$$
\underset{n \rightarrow \infty}{\lim \sup _{n}} S_{n}\left(2 B_{n}^{2} \log \log B_{n}\right)^{-1 / 2}=1 \text { a.e. }
$$

1.4. Salem and Zygmund [1] , [2] showed that the conclusion of 1.2 holds for $\Omega=T$, the circle group, for $P$, normalized Haar measured on $T$ and $\left\{f_{k}\right\}_{k=1}^{\infty}$, a sequence of trigonometric functions defined by each $t \in T$ by

$$
f_{k}(t)=a_{k} \cos \left(n_{k} t\right)+b_{k} \sin \left(n_{k} t\right)
$$

where $a_{k}$ and $b_{k}$ are real numbers and $\left\{n_{k}\right\}_{k=1}^{\infty}$ is a Hadamard sequence of positive integers (that is, $n_{k+1} / n_{k} \geqslant q$ for some real number $q>1$ and all $k$ ). Note that the $f_{k}$ 's are not independent. After preliminary results were obtained by Salem and Zygmund [3] and Erdös and Gál [1], the corresponding theorem 1.3 was proved by Mary Weiss [1].

1.5. We now give a formulation of the abstract problem. Throughout this paper, unless otherwise specified, the symbol $G$ will be used to denote an arbitrary infinite compact abelian group. $\lambda$ will denote normalized Haar measure on $\boldsymbol{G}$ while the character group of $G$, which will be taken to be a multiplicative group, will be denoted by $\hat{G}$. Let $\Upsilon=\left\{X_{n}\right\}_{n=1}^{\infty}$ be a sequence of nonempty, pairwise disjoint, finite subsets of $\hat{G}$ which are symmetric (that is, $\chi \in X_{n}$ implies that $\chi^{-1} \in X_{n}$ ). Suppose that, for a given countably infinite, symmetric subset $Q$ of $\bigcup_{n=1}^{\infty} X_{n}$, there is defined a complex-valued function $u$ on $Q$ which is symmetric (that is, $\left.u\left(\chi^{-1}\right)=\overline{u(\chi)}\right)$. We put $Q_{n}=Q \cap X_{n}$ and set

$$
S_{n}=\sum_{k=1}^{n} \sum_{\chi \in Q_{k}} u(\chi) \chi, \quad B_{n}=\left(\sum_{k=1}^{n} \sum_{\chi \in Q_{k}}|u(\chi)|^{2}\right)^{1 / 2},
$$


and

$$
M_{n}=\max _{k=1,2, \ldots, n} \max _{\chi \in Q_{k}}|u(\chi)| \text {. }
$$

Obviously $S_{n}, B_{n}$ and $M_{n}$ depend on $\gamma, Q$ and $u$ but in most instances there will be no need to indicate this explicitly.

We investigate the behaviour of quadruples $(G, \gamma, Q, u)$ in relation to the Gaussian law and the law of the iterated logarithm. Specifically, we suppose that $R$ is a lacunary property of subsets of $\hat{G}$ and we set

$$
H_{R, G}=\left\{(G, \gamma, Q, u): Q=P \cup P^{-1}, P \text { has property } R\right\}
$$

where $P^{-1}=\left\{\chi^{-1}: \chi \in P\right\}$. It is then natural to ask for what properties $R$ do the conclusions of 1.2 and 1.3 hold in the case when $\Omega=G, P=\lambda,(G, \gamma, Q, u)$ $\in H_{R, G}$ and $f_{k}=\Sigma_{\chi \in Q_{k}} u(\chi) \chi$. We will show that the conclusion of 1.2 holds for a certain subclass of Stečin sets but fails for other such sets and a modified law of the iterated logarithm holds to varying degrees for different subclasses of Steckin sets.

The sets examined are defined in $\$ 2$ and certain properties are proved which are intimately connected with the quasi independence of the sets in question. In $\S 3$, we prove the Gaussian law for a certain lacunary property and show that it fails to hold for other properties. In $\S 4$, we introduce parts of the theory of dyadic representations of positive integers to obtain an estimate of the $L_{p}$ norm of functions each of whose values at a given point is the supremum of the values of a finite number of trigonometric polynomials. Finally in $\$ 5$, we utilize the results obtained in $\S \S 2$ and 4 to prove a law of the iterated logarithm for lacunary sets of characters.

1.6. Throughout this paper, we denote the set of integers by $Z$, the positive integers by $N$, the real numbers by $R$ and the complex numbers by $K$. We use the symbols $[a, b],] a, b[,[a, b[$ and $] a, b]$ for $a, b \in R$ and $a \leqslant b$ to denote the appropriate closed interval, open interval and half open intervals of real numbers. For certain subsections which will be indicated, we will use this notation to denote intervals of integers. For $S$ any set, we write card $S$ for the cardinality of $S$. For $f$, a real-valued function, and $a \in R$, we write $\{f>a\}$ for the set $\{t: f(t)>a\}$. For $f$, a nonzero complex-valued function, we denote by $f^{-1}$ the function $f^{-1}$ : $t \rightarrow(f(t))^{-1}$. Note that for $\chi \in G, \chi^{-1}$ is the group inverse of $\chi$. For $P \subset G$, we set $P^{-1}=\left\{\chi^{-1}: \chi \in P\right\}$. The end of a proof will be denoted by the symbol $\square$.

\section{2}

2.1. We denote the identity element of $\hat{G}$ by the symbol 1 . For each $P$ $\subset \hat{G}$, we set

$$
P(2)=\left\{\chi \in P: \chi^{2}=1\right\} \text { and } P(>2)=\left\{\chi \in P: \chi^{2} \neq 1\right\} .
$$


The definitions which follow stem in part from Stečkin's work [1] and are in part similar to those appearing in $\$ 10$ of Edwards, Hewitt and Ross [2]. For $n \in N$ and $P \subset \hat{G}$, we let $S_{2}(P, n)$ be the set of all functions $g$ defined on $P$ which take values in $\{-2,-1,0,1,2\}$ and have the properties that $\Sigma_{\chi \in P}|g(\chi)|=n$ and $g(\chi) \in\{-1,0,1\}$ for all $\chi \in P(2)$. We let $S_{1}(P, n)$ consist of all functions in $S_{2}(P, n)$ which take values in $\{-1,0,1\}$. For $\phi \in \hat{G}$ and $j=1$ or 2 , we set

$$
S_{j}(P, n, \phi)=\left\{g \in S_{j}(P, n): \prod_{x \in P} \chi^{g(x)}=\phi\right\} \text {. }
$$

DEFinitions. Let $P$ be a nonempty subset of $G$ such that $P(>2) \cap P(>2)^{-1}$ $=\varnothing$ and let $j=1$ or 2 . Then we say that $P$ has property $\left(R_{j}\right)$ if there exists a positive number $\omega$ such that card $S_{j}(P, n, 1) \leqslant \omega^{n}$ for all $n \in N$. The set of all $\omega \geqslant 1$ which have this property clearly has a minimum element called the $\boldsymbol{R}_{\boldsymbol{j}}$ bound for $P$. We will say that $P$ is a $j$-dissociate set if, for each $n \in N$, the set $S_{j}(P, n, 1)$ is empty. If $P$ is the finite union of sets, each of which has the property $\left(R_{j}\right)$, then we will say that $P$ is a $j$-Steckin set.

2.2. Comment. Since we have $S_{1}(P, n, 1) \subset S_{2}(P, n, 1)$ for all $n \in N$, it is clear that a set having property $\left(R_{2}\right)$ has property $\left(R_{1}\right)$, that a 2-dissociate set is a 1-dissociate set and that a 2-Stečkin set is a 1-Stečkin set. However a 1-dissociate set may fail to be a 2 -Stečkin set. An example of such a set will be given in 3.3.

2.3. The next two lemmas yield the properties critical for the theorems that follow.

LEMmA. Suppose that $P \subset \hat{G}$ has the property $\left(R_{2}\right)$ with $R_{2}$-bound equal to $\omega$ and let $\xi$ be any positive number less than $\omega^{-1}$. Further let $V$ be a nonempty finite subset of $P$ and $f$ a complex-valued function defined on $V \times\{-2$, $-1,0,1,2\}$ such that:

(i) if $\chi \in V(2)$, then $f(\chi, k)$ is nonzero only if $k \in\{-1,0,1\}$; and

(ii) for all $\chi \in V$ and $k \in\{-2,-1,0,1,2\},|f(\chi, k)| \leqslant \xi^{|k|}|f(\chi, 0)|$. Then we have

$$
\begin{array}{r}
\left|\int_{G} \prod_{\chi \in V}\left(\sum_{k=-2}^{2} f(\chi, k) \chi^{k}\right) d \lambda-\prod_{\chi \in V} f(x, 0)\right| \\
\leqslant \xi \omega(1-\xi \omega)^{-1} \prod_{\chi \in V}|f(\chi, 0)| .
\end{array}
$$

Proof. For convenience, set $A=\{-2,-1,0,1,2\}$. We let $B$ be the set of all nonzero fi zctions from $V$ into $A$ and we set

$$
C=\left\{g \in B: \prod_{x \in V} \chi^{g(x)}=1\right\} \text {. }
$$


It is clear that

$$
\begin{aligned}
\prod_{\chi \in V} & \left(\sum_{k \in A} f(x, k) \chi^{k}\right) \\
& =\prod_{\chi \in V} f(x, 0)+\sum_{g \in B} \prod_{x \in V} f(x, g(x)) \prod_{\chi \in V} \chi^{g(x)} .
\end{aligned}
$$

Recalling that $\int_{G} \chi d \lambda=\delta_{\chi 1}$ and integrating both sides of (2.1), we obtain

$$
\int_{G} \prod_{\chi \in V}\left(\sum_{k \in A} f(\chi, k) \chi^{k}\right) d \lambda=\prod_{x \in V} f(\chi, 0)+\sum_{g \in C} \prod_{\chi \in V} f(\chi, g(\chi)) .
$$

To prove (iii), it is sufficient to show that

$$
\sum_{g \in C} \prod_{\chi \in V}|f(\chi, g(x))| \leqslant \xi \omega(1-\xi \omega)^{-1} \prod_{\chi \in V}|f(x, 0)| .
$$

By condition (i), a summand in the left side of (2.2) is nonzero only if $g(x) \in$ $\{-1,0,1\}$ for $\chi \in V(2)$. A function $g \in C$ which has this property belongs to $S_{2}\left(V, \Sigma_{\chi \in V} g(X), 1\right)$. Noting condition (ii) and the fact that $\Sigma_{\chi \in V}|g(\chi)|=n$ for $g \in S_{2}(V, n, 1)$, we obtain

$$
\begin{aligned}
\sum_{g \in C} \prod_{x \in V}|f(\chi, g(x))| & \leqslant \sum_{n=1}^{\infty} \sum_{g \in S_{2}(V, n, 1)} \prod_{x \in V}|f(\chi, g(x))| \\
& \leqslant \prod_{\chi \in V}|f(\chi, 0)| \sum_{n=1}^{\infty} \sum_{g \in S_{2}(V, n, 1)} \xi^{n} \\
& =\prod_{\chi \in V}|f(\chi, 0)| \sum_{n=1}^{\infty} \operatorname{card} S_{2}(V, n, 1) \xi^{n} .
\end{aligned}
$$

Since we have $S_{2}(V, n, 1) \leqslant \operatorname{card} S_{2}(P, n, 1) \leqslant \omega^{n}$, an easy calculation yields (2.2). ㅁ

\subsection{A similar argument yields the following:}

LEMMA. Suppose that $P \subset \hat{G}$ has the property $\left(R_{1}\right)$ with $R_{1}$-bound equal to $\omega$ and let $\xi$ be a positive number less than $\omega^{-1}$. Further let $V$ be a finite subset of $P$ and $f$ a complex-valued function defined on $V \times\{-1,0,1\}$ such that for all $\chi \in V$ and $k \in\{-1,0,1\}$, we have

$$
|f(\chi, k)| \leqslant \xi^{|k|}|f(\chi, 0)| .
$$

Then we have the following estimate:

$$
\begin{array}{r}
\left|\int_{G} \prod_{x \in V}\left(\sum_{k \in\{-1,0,1\}} f(x, k) \chi^{k}\right) d \lambda-\prod_{x \in V} f(x, 0)\right| \\
\leqslant \xi \omega(1-\xi \omega)^{-1} \prod_{x \in V}|f(x, 0)| .
\end{array}
$$


2.5. For the remainder of this section, we will consider nonempty sets $P \subset \hat{G}$ for which $P(>2) \cap P(>2)^{-1}=\varnothing$. We let $V$ be any nonempty finite subset of $P$ and we put $W=V \cup V^{-1}$. Furthermore we let $u$ be a symmetric function defined on $W$ and we set

$$
S=\sum_{\chi \in W} u(\chi) \chi, \quad B=\left(\sum_{\chi \in W}|u(\chi)|^{2}\right)^{1 / 2} \quad \text { and } \quad M=\max _{\chi \in W}|u(\chi)| .
$$

There will be no necessity to indicate explicitly the dependence of $S, B$ and $M$ on $W$ and $n$. We show that sets having the properties $\left(R_{1}\right)$ or $\left(R_{2}\right)$ satisfy certain integral estimates critical to the proof that such sets have the iterated logarithm property and the Gaussian property.

2.6. THEOREM. (a) Suppose that $P$ has property $\left(R_{2}\right)$ with $R_{2}$-bound equal to $\omega$. Let $\epsilon$ be any positive number less than 12 and suppose that $\theta$ is any complex number such that

$$
48 \omega|\theta| M<\epsilon
$$

Then the following is true:

$$
\int_{G} \exp (\theta S) d \lambda=\left(1+\eta_{1}\right) \exp \left(1 / 2 \theta^{2} B^{2}\left(1+\eta_{2}\right)\right)+\eta_{3}|\theta|^{2} B^{2} \exp \left(3|\theta|^{2} B^{2}\right)
$$

where $\eta_{1}, \eta_{2}$ and $\eta_{3}$ are complex numbers each of which has absolute value at most equal to $\epsilon$.

(b) If, in addition, $\theta$ is positive, then we have

$$
\int_{G} \exp (\theta S) d \lambda=\left(1+\eta_{1}\right) \exp \left(1 / 2 \theta^{2} B^{2}\left(1+\eta_{2}\right)\right)
$$

where $\eta_{1}$ and $\eta_{2}$ are real numbers, each of which has absolute value at most equal to $\epsilon$.

2.7. TheOREM. Suppose that $P$ has property $\left(R_{1}\right)$ with $R_{1}$-bound equal to $\omega$. Let $\epsilon$ be any positive number less than 1 and suppose that $\theta$ is a positive number such that $4 \omega \theta M<\epsilon$. We have

$$
(1-\epsilon) \leqslant \int_{G} \exp (\theta S) d \lambda \leqslant(1+\epsilon) \exp \left((1+\epsilon) \theta^{2} B^{2}\right) .
$$

2.8. Proof of 2.6(a). For $|z|<1 / 2$, we have, from the power series of the $\log$ function,

$$
\exp z=\left(1+z+1 / 2 z^{2}\right) \exp h(z) \quad \text { where }|h(z)| \leqslant 3|z|^{3} .
$$

Noting that $2|\theta||u(\chi)|<2|\theta| M<\epsilon(24 \omega)^{-1}<1 / 2$, we write

$$
\begin{aligned}
\exp (\theta S) & =\prod_{\chi \in V(2)} \exp (\theta u(\chi) \chi) \prod_{\chi \in V(>2)} \exp \left(\theta u(\chi) \chi+\theta u\left(\chi^{-1}\right) \chi^{-1}\right) \\
& =\Delta_{(2)} \Delta_{(>2)} \Phi_{(2)} \Phi_{(>2)}
\end{aligned}
$$


where

$$
\begin{aligned}
\Delta_{(2)} & =\prod_{\chi \in V(2)}\left(1+\theta u(\chi) \chi+1 / 2 \theta^{2}(u(\chi))^{2}\right), \\
\Delta_{(>2)} & =\prod_{\chi \in V(>2)}\left(1+\theta u(\chi) \chi+\theta u\left(\chi^{-1}\right) \chi^{-1}+1 / 2\left(\theta u(\chi) \chi+\theta u\left(\chi^{-1}\right) \chi^{-1}\right)^{2}\right), \\
\Phi_{(2)} & =\prod_{\chi \in V(2)} \exp h(\theta u(\chi) \chi)
\end{aligned}
$$

and

$$
\Phi_{(>2)}=\prod_{\chi \in V(>2)} \exp h\left(\theta u(\chi) \chi+\theta u\left(\chi^{-1}\right) \chi^{-1}\right)
$$

From (2.6), we conclude that

$$
\begin{aligned}
& \left|\sum_{\chi \in V(2)} h(\theta u(\chi) \chi)+\sum_{\chi \in V(>2)} h\left(\theta u(\chi) x+\theta u\left(\chi^{-1}\right) \chi^{-1}\right)\right| \\
& \quad \leqslant \sum_{\chi \in V(2)} 3|\theta u(\chi) \chi|^{3}+\sum_{\chi \in V(>2)} 3\left|\theta u(\chi) \chi+\theta u\left(\chi^{-1}\right) \chi^{-1}\right|^{3} \\
& \quad \leqslant 3 \sum_{x \in V(2)}|\theta|^{3} M|u(\chi)|^{2}+24 \sum_{x \in V(>2)}|\theta|^{3} M|u(\chi)|^{2} \\
& \quad \leqslant 12|\theta|^{3} M\left(\sum_{x \in V(2)}|u(\chi)|^{2}+2 \sum_{x \in V(>2)}|u(\chi)|^{2}\right) .
\end{aligned}
$$

Since we are given that $48|\theta| M<\epsilon$ and since it is readily observed that $B^{2}=$ $\Sigma_{\chi \in V(2)}|u(\chi)|^{2}+2 \Sigma_{\chi \in V(>2)}|u(\chi)|^{2}$, we conclude that

$$
\left|\sum_{\chi \in V(2)} h(\theta u(\chi) \chi)+\sum_{\chi \in V(>2)} h\left(\theta u(\chi) \chi+\theta u\left(\chi^{-1}\right) \chi^{-1}\right)\right| \leqslant(\epsilon / 4)|\theta|^{2} B^{2} .
$$

Since $|\exp z-1| \leqslant|z| \exp |z|$ for all $z$, it follows that

$$
\left|\Phi_{(2)}(t) \Phi_{(>2)}(t)-1\right| \leqslant(\epsilon / 4)|\theta|^{2} B^{2} \exp \left((\epsilon / 4)|\theta|^{2} B^{2}\right) \text { for all } t \in G \text {. }
$$

We now estimate $\int_{G} \Delta_{(2)} \Delta_{(>2)} d \lambda$. From (2.8), we write

and

$$
\Delta_{(2)}=\prod_{\chi \in V(2)}\left(1+\theta u(\chi) \chi+1 / 2 \theta^{2}|u(\chi)|^{2}\right)
$$

$$
\begin{aligned}
\Delta_{(>2)}=\prod_{\chi \in V(>2)}\left(1+\theta^{2}|u(\chi)|^{2}\right. & +\theta u(\chi) \chi+\theta u\left(\chi^{-1}\right) \chi^{-1} \\
& \left.+1 / 2 \theta^{2} u(\chi)^{2} \chi^{2}+1 / 2 \theta^{2} u\left(\chi^{-1}\right)^{2} \chi^{-2}\right)
\end{aligned}
$$

We estimate $\int_{G} \Delta_{(2)} \Delta_{(>2)} d \lambda$ by applying 2.3 . We set $\xi=2|\theta| M$ and define $f$ in the appropriate manner. It is clear that condition (i) of the lemma holds for our function $f$ while condition (ii) is seen to hold for our choice of $f$ and $\xi$ once we 
observe that

$$
\left.\left|1+\theta^{2}\right| u(x)\right|^{2} \mid \geqslant 1-\theta^{2} M^{2}>1 / 2 \quad(\text { see }(2.4)) .
$$

We obtain

$$
\begin{aligned}
\int_{G} \Delta_{(2)} \Delta_{(>2)} d \lambda= & \left(1+\eta_{1}\right) \prod_{\chi \in V(2)}\left(1+\frac{1 / 2}{2}|u(\chi)|^{2}\right) \\
& \times \prod_{\chi \in V(>2)}\left(1+\theta^{2}|u(x)|^{2}\right)
\end{aligned}
$$

where

$$
\begin{aligned}
\left|\eta_{1}\right| & \leqslant \xi \omega(1-\xi \omega)^{-1} \\
& =2|\theta| M \omega(1-2|\theta| M \omega)^{-1} \\
& <\epsilon \quad(\text { since } 2|\theta| M \omega<\epsilon / 24<1 / 2 \text { by }(2.4)) .
\end{aligned}
$$

For $|u|<1 / 2$, we have the formula

$$
1+u=\exp (u+k(u)) \quad \text { where }|k(u)| \leqslant 2|u|^{2} .
$$

We rewrite (2.11) as

$$
\begin{aligned}
\int_{G} \Delta_{(2)} \Delta_{(>2)} d \lambda= & \left(1+\eta_{1}\right) \prod_{\chi \in V(2)} \exp \left(1 / 2 \theta^{2}|u(x)|^{2}+k\left(1 / 2 \theta^{2}|u(x)|^{2}\right)\right) \\
& \times \prod_{x \in V(>2)} \exp \left(\theta^{2}|u(x)|^{2}+k\left(\theta^{2}|u(x)|^{2}\right)\right) \\
& =\left(1+\eta_{1}\right) \exp \left(1 / 2 \theta^{2} B^{2}\left(1+\eta_{2}\right)\right)
\end{aligned}
$$

where

$$
\eta_{2}=2\left(\theta^{2} B^{2}\right)^{-1}\left(\sum_{x \in V(2)} k\left(1 / 2 \theta^{2}|u(x)|^{2}\right)+\sum_{x \in V(>2)} k\left(\theta^{2}|u(x)|^{2}\right)\right) .
$$

We have

$$
\begin{aligned}
\left|\eta_{2}\right| & \leqslant 4\left(|\theta|^{2} B^{2}\right)^{-1}\left(\sum_{x \in V(2)} 1 / 4|\theta|^{4}|u(x)|^{4}+\sum_{x \in V(>2)}|\theta|^{4}|u(x)|^{4}\right) \\
& \leqslant|\theta|^{2} B^{-2}\left(\sum_{x \in V(2)}|u(x)|^{4}+2 \sum_{x \in V(>2)}|u(x)|^{4}\right) \\
& \leqslant|\theta|^{2} M^{2} B^{-2}\left(\sum_{x \in V(2)}|u(x)|^{2}+2 \sum_{x \in V(>2)}|u(x)|^{2}\right) \\
& =|\theta|^{2} M^{2}<\epsilon / 2 .
\end{aligned}
$$

From (2.7), we have

$$
\begin{aligned}
\int_{G} \exp (\theta S) d \lambda & =\int_{G} \Delta_{(2)} \Delta_{(>2)} \Phi_{(2)} \Phi_{(>2)} d \lambda \\
& =\int_{G} \Delta_{(2)} \Delta_{(>2)} d \lambda-\int_{G}\left(1-\Phi_{(2)} \Phi_{(>2)} \Delta_{(2)} \Delta_{(>2)} d \lambda .\right.
\end{aligned}
$$


In view of (2.10) and (2.12), it remains to show that

$$
\int_{G}\left|\Delta_{(2)} \Delta_{(>2)}\right| d \lambda<4 \exp \left(3|\theta|^{2} B^{2}\right) .
$$

By Hölder's inequality, it is sufficient to show that

$$
\int_{G}\left|\Delta_{(2)} \Delta_{(>2)}\right|^{2} d \lambda<16 \exp \left(6|\theta|^{2} B^{2}\right)
$$

Noting that $u(\chi) \chi+u\left(\chi^{-1}\right) \chi^{-1}$ is real for $\chi \in V(>2)$ and $u$ symmetric, we obtain for $\chi \in V(>2)$ after a routine calculation using the estimate $|\theta||u(\chi)| \leqslant|\theta| M<$ $1 / 4$,

$$
\begin{aligned}
\mid 1+\theta(u(\chi) \chi & \left.+u\left(\chi^{-1}\right) \chi^{-1}\right)+1 /\left.2 \theta^{2}\left(u(\chi) \chi+u\left(\chi^{-1}\right) \chi^{-1}\right)^{2}\right|^{2} \\
& \leqslant 1+(\theta+\bar{\theta})\left(u(\chi) \chi+u\left(\chi^{-1}\right) \chi^{-1}\right)+12|\theta|^{2}|u(\chi)|^{2} .
\end{aligned}
$$

A similar calculation yields for $\chi \in V(2)$ :

$$
\left.\left.\left|1+\theta u(\chi) \chi+1 / 2 \theta^{2}\right| u(\chi)\right|^{2}\right|^{2} \leqslant 1+(\theta+\bar{\theta}) u(\chi) \chi+6|\theta|^{2}|u(\chi)|^{2} .
$$

Noting (2.8), we combine these estimates to obtain

$$
\begin{aligned}
\left|\Delta_{(2)} \Delta_{(>2)}\right|^{2} \leqslant & \prod_{x \in V(2)}\left(1+6|\theta|^{2}|u(x)|^{2}+(\theta+\bar{\theta}) u(x) \chi\right) \\
& \times \prod_{x \in V(>2)}\left(1+12|\theta|^{2}|u(x)|^{2}+(\theta+\bar{\theta})\left(u(x) \chi+u\left(x^{-1}\right) \chi^{-1}\right)\right) .
\end{aligned}
$$

We apply 2.3 again for $\xi=|\theta| M$ to obtain

$$
\begin{aligned}
\int_{G}\left|\Delta_{(2)} \Delta_{(>2)}\right|^{2} d \lambda \leqslant & \left(1+\eta^{1}\right) \prod_{\chi \in V(2)}\left(1+6 \theta^{2}|u(\chi)|^{2}\right) \\
& \times \prod_{x \in V(>2)}\left(1+12 \theta^{2}|u(\chi)|^{2}\right)
\end{aligned}
$$

where $\eta^{1}$ is necessarily real and $\left|\eta^{1}\right| \leqslant \xi \omega(1-\xi \omega)^{-1} \leqslant \omega|\theta| M(1-\omega|\theta| M)^{-1}<$ 1.

Now since $1+x \leqslant \exp x$ for all $x>0$, we have

$$
\begin{aligned}
\int_{G}\left|\Delta_{(2)} \Delta_{(>2)}\right|^{2} d \lambda & \leqslant 2 \prod_{x \in V(2)} \exp \left(6|\theta|^{2}|u(x)|^{2}\right) \prod_{x \in V(>2)} \exp \left(12|\theta|^{2}|u(x)|^{2}\right) \\
& \leqslant 2 \exp \left(6|\theta|^{2} B^{2}\right) .
\end{aligned}
$$

Thus we have shown (2.13) and our proof is complete.

2.9. Proof of 2.6(b). We note that, if $z$ is real and $|z|>1 / 2$, then, in (2.6), the function $h(z)$ is real valued and $1+z+1 / 2 z^{2}>1 / 2>0$. It follows from (2.8) and (2.9) that $\exp \left(-(\epsilon / 4) \theta^{2} B^{2}\right) \leqslant \Phi_{(2)} \Phi_{(>2)} \leqslant \exp \left((\epsilon / 4) \theta^{2} B^{2}\right)$. Since $\Delta_{(2)}$ and $\Delta_{(>2)}$ are greater than 0 , we obtain 


$$
\begin{aligned}
\exp \left(-(\epsilon / 4) \theta^{2} B^{2}\right) \int_{G} \Delta_{(2)} \Delta_{(>2)} d \lambda & \leqslant \int_{G} \Phi_{(2)} \Phi_{(>2)} \Delta_{(2)} \Delta_{(>2)} d \lambda \\
& \leqslant \exp \left((\epsilon / 4) \theta^{2} B^{2}\right) \int_{G} \Delta_{(2)} \Delta_{(>2)} d \lambda .
\end{aligned}
$$

The desired result is obtained by combining (2.7) with (2.12) and (2.14).

2.10. Proof of 2.7. For $|z|<1 / 2$, we have

$$
\exp z=(1+z) \exp \left(1 / 2(1+j(z)) z^{2}\right) \quad \text { where }|j(z)|<|z| .
$$

Note that if $z$ is real then so is $j(z)$. Noting that $2 \theta|u(x)| \leqslant 2 \theta M<1 / 2$, we write

$$
\begin{aligned}
& \exp (\theta S)=\prod_{\chi \in V(2)} \exp (\theta u(\chi) \chi) \prod_{\chi \in V(>2)} \exp \left(\theta u(x) \chi+\theta u\left(\chi^{-1}\right) \chi^{-1}\right) \\
& =\prod_{x \in V(2)}(1+\theta u(x) x) \prod_{x \in V(>2)}\left(1+\theta u(x) x+\theta u\left(x^{-1}\right) x^{-1}\right) \\
& \times \prod_{x \in V(2)} \exp \left(\frac{1}{2} /(1+j(\theta u(x) x)) \theta^{2} u(x)^{2}\right) \\
& \times \prod_{x \in V(>2)} \exp \left(1 / 2\left(1+j\left(\theta u(x) x+\theta u\left(\chi^{-1}\right) x^{-1}\right)\right)\right. \\
& \left.\times \theta^{2}\left(u(x) x+u\left(x^{-1}\right) x^{-1}\right)^{2}\right) .
\end{aligned}
$$

Note that $\theta u(\chi) \chi+\theta u\left(\chi^{-1}\right) \chi^{-1}$ for $\chi \in V(>2)$ and $u(\chi)$ for $\chi \in V(2)$ are real valued and have absolute value less than or equal to $2 \theta|u(\chi)| \leqslant 2 \theta M$. We use the bound on $j(z)$ given in (2.15) and the fact that $B^{2}=\Sigma_{\chi \in V(2)}|u(\chi)|^{2}+$ $2 \Sigma_{x \in V(>2)}|u(x)|^{2}$ to obtain

$$
\begin{aligned}
& 1 \leqslant \prod_{\chi \in V(2)} \exp \left(1 / 2(1+j(\theta u(\chi) x)) \theta^{2} u(\chi)^{2}\right) \\
& \quad \times \prod_{\chi \in V(>2)} \exp \left(1 / 2\left(1+j\left(\theta u(\chi) \chi+\theta u\left(\chi^{-1}\right) \chi^{-1}\right)\right)\right. \\
& \left.\quad \times \theta^{2}\left(u(x) \chi+u\left(\chi^{-1}\right) \chi^{-1}\right)^{2}\right) \\
& \leqslant \prod_{\chi \in V(2)} \exp \left(1 / 2(1+\epsilon) \theta^{2}|u(\chi)|^{2}\right) \\
& \quad \times \prod_{x \in V(>2)} \exp \left(2(1+\epsilon) \theta^{2} u(\chi) \chi^{2}\right) \\
& \leqslant \exp \left((1+\epsilon) \theta^{2} B^{2}\right) .
\end{aligned}
$$

Since $1+\theta u(x) \chi \geqslant 3 / 4>0$ for $\chi \in V(2)$ and $1+\theta u(\chi) x+\theta u\left(\chi^{-1}\right) x^{-1} \geqslant 1 / 2>0$ for $\chi \in V(>2)$, we infer from (2.16) and (2.17) that

where

$$
\Delta \leqslant \exp (\theta S) \leqslant \exp \left((1+\epsilon) \theta^{2} B^{2}\right) \Delta
$$




$$
\Delta=\prod_{\chi \in V(2)}(1+\theta u(\chi) \chi) \prod_{\chi \in V(>2)}\left(1+\theta u(\chi) \chi+\theta u\left(\chi^{-1}\right) \chi^{-1}\right) .
$$

It remains to observe that 2.4 can be used to evaluate $\int_{G} \Delta d \lambda$. We obtain $\int_{G} \Delta d \lambda=1+\eta$ where $|\eta| \leqslant \theta M \omega(1-\theta M \omega)^{-1}$.

2.11. An upper bound for $\int_{G} \exp (\theta S) d \lambda$ is easily computed using Hölder's inequality for the case in which $P$ is a 2 -Stečkin set and $\theta>0$.

COROLlary. Suppose that $P$ is a 2-Stečkin set which is the union of $m$ sets $P_{1}, P_{2}, \ldots, P_{m}$, each $P_{k}$ having the property $\left(R_{2}\right)$ with $R_{2}$-bound equal to $\omega_{k}$. Let $\omega=\max _{k=1,2, \ldots, m} \omega_{k}$. Let $\epsilon$ be any positive number less than 12 and suppose that $\theta$ is a positive number such that $48 \omega M \theta M<\epsilon$. It follows that

$$
\int_{G} \exp (\theta S) d \lambda \leqslant(1+\epsilon) \exp \left((m / 2) \theta^{2} B^{2}(1+\epsilon)\right)
$$

Proof. We write $V=\bigcup_{k=1}^{m} V_{k}$ where $V_{k} \subset P_{k}$. Without loss of generality, we may suppose that this union is a disjoint union of nonempty sets. Setting $W_{k}=V_{k} \cup V_{k}^{-1}$, we define

$$
S_{k}=\sum_{\chi \in W_{k}} u(\chi) \chi, \quad B_{k}=\left(\sum_{\chi \in W_{k}}|u(\chi)|^{2}\right)^{1 / 2} \text { and } M_{k}=\max _{\chi \in W_{k}}|u(\chi)| .
$$

It is clear that

$$
S=\sum_{k=1}^{m} S_{k}, \quad B=\left(\sum_{k=1}^{m} B_{k}^{2}\right)^{1 / 2} \text { and } M=\max _{k=1, \ldots, m} M_{k} .
$$

We apply $2.6(\mathrm{~b})$ to obtain

$$
\int_{G} \exp \left(m \theta B_{k}\right) d \lambda \leqslant(1+\epsilon) \exp \left(1 / 2 m^{2} \theta^{2} B_{k}^{2}(1+\epsilon)\right)
$$

We rewrite this as

$$
\left\|\exp \left(m \theta S_{k}\right)\right\|_{1}^{1 / m} \leqslant(1+\epsilon)^{1 / m} \exp \left(1 / 2 m \theta^{2} B_{k}^{2}(1+\epsilon)\right) .
$$

Since we have $\exp (\theta S)=\Pi_{k=1}^{m}\left(\exp \left(m \theta S_{k}\right)\right)^{1 / m}$, we can apply (12.5) of Hewitt and Ross, Vol. I, to obtain

$$
\int_{G} \exp (\theta S) d \lambda \leqslant \prod_{k=1}^{m}\left\|\exp \left(m \theta S_{k}\right)\right\|_{1}^{1 / m}
$$

Noting (2.19) and (2.20), we conclude that (2.18) holds. $\square$

2.12. A similar argument yields the corresponding corollary to 2.7 .

COROLlary. Suppose that $P$ is a 1 Steckkin set which is the union of $m$ sets $P_{1}, P_{2}, \ldots, P_{m}$, each $P_{k}$ having property $\left(R_{1}\right)$ with $R_{1}$-bound equal to $\omega_{k}$. Put $\omega=\max _{k=1,2, \ldots, m} \omega_{k}$. Let $\epsilon$ be any positive number less than 1 and suppose 
that $\theta$ is a positive number such that $4 \omega M \theta M<\epsilon$. We have

$$
\int_{G} \exp (\theta S) d \lambda \leqslant(1+\epsilon) \exp \left((1+\epsilon) m \theta^{2} B^{2}\right) .
$$

2.13. The inequalities (2.18) and (2.21) are those used to prove our version of the law of iterated logarithm. It will prove convenient to formulate the following definition.

Definition. A nonempty set of characters $P$ such that $P(>2) \cap P(>2)^{-1}$ $=\varnothing$ is said to be $\Phi$-bounded by a positive number $\phi$ if there exist positive numbers $k_{1}, k_{2}$ and $k_{3}$, such that the following property holds. Given a finite nonempty subset $V$ of $P$, a symmetric function $u$ defined on $W=V \cup V^{-1}$ and a positive number $\epsilon$ less than $k_{1}$, then if $\theta$ is a positive number such that $k_{2} \theta M<$ $\epsilon$, we have

$$
\int_{G} \exp (\theta S) d \lambda \leqslant k_{3} \exp \left(\phi(1+\epsilon) \theta^{2} B^{2}\right) .
$$

2.14. We reformulate 2.11 and 2.12 as:

THEOREM. (a) A 22Stečkin set which is the union of $m$ sets having the property $\left(R_{2}\right)$ is $\Phi$-bounded by $1 / 2 \mathrm{~m}$.

(b) $A 1$ Steckin set which is the union of $m$ sets having the property $\left(R_{1}\right)$ is $\Phi$-bounded by $m$.

2.15. We have the following easy consequences of the definition.

THEOREM. Suppose that $P$ is $\Phi$-bounded by $\phi$. Let $V$ be a finite nonempty subset of $P$ and suppose that $u$ is a symmetric function defined on $W=V \cup V^{-1}$. Let $\epsilon$ be a positive number such that $k_{2} M<\epsilon$. Then for any real number $y$, we have

$$
\lambda\{S \geqslant y\} \leqslant k_{3} \exp \left(\phi(1+\epsilon) \theta^{2} B^{2}-\theta y\right) .
$$

Proof. The proof follows readily from the inequality:

$$
\lambda\{S \geqslant y\} \exp (\theta y) \leqslant \int_{G} \exp (\theta S) d \lambda .
$$

3.1. We now prove the Gaussian law for sets having the property $\left(R_{2}\right)$ and show that it can fail for the union of two such sets and for sets having the property $\left(R_{1}\right)$.

THEOREM. Let $\Upsilon=\left\{X_{n}\right\}_{n=1}^{\infty}$ be as described in 1.5. Suppose that $P \subset$ $\bigcup_{n=1}^{\infty} X_{n}$ is a countably infinite set having the property $\left(R_{2}\right)$ with $R_{2}$-bound equal to $\omega$. Let $u$ be a symmetric function defined on $Q=P \cup P^{-1}$ and suppose 
that (i) $B_{n} \rightarrow \infty$, and (ii) $M_{n} B_{n}^{-1}=o$ (1) as $n \rightarrow \infty$. Then we have

$$
\lim _{n \rightarrow \infty} \lambda\left\{S_{n}<y B_{n}\right\}=(2 \pi)^{-1 / 2} \int_{-\infty}^{y} \exp \left(-1 / 2 t^{2}\right) d t=G_{1}(y)
$$

for all $y \in R$.

Proof. Setting $F_{n}: y \rightarrow \lambda\left\{S_{n}<y B_{n}\right\}$, we define the characteristic function $f_{n}$ of $F_{n}$ by the formula:

$$
f_{n}(u)=\int_{-\infty}^{\infty} \exp (i u y) d F_{n}(y)=\int_{G} \exp \left(i u S_{n} B_{n}^{-1}\right) d \lambda .
$$

Since $\exp \left(-1 / 2 u^{2}\right)$ is the characteristic function of $G_{1}(y)$, in order to prove our theorem, it suffices to show that, for any $A>0, \lim _{n \rightarrow \infty} f_{n}(u)=\exp \left(-1 / 2 u^{2}\right)$ uniformly for $|u|<A$ (see Gnedenko [1, §38]). Let $\epsilon$ be any positive number less than 12. Choose $n_{\epsilon} \in N$ such that $48 \omega A M_{n} B_{n}^{-1}<\epsilon$ for all $n>n_{\epsilon}$. For each $n>n_{\epsilon}$ and each $u$ such that $|u|<A$, we apply 2.6(a) to the set $V=$ $\bigcup_{k=1}^{n} P_{k}$ where $P_{k}=X_{k} \cap P$, for $\epsilon$ as given and for $\theta=i u B_{n}^{-1}$. We obtain

$$
f_{n}(u)=\left(1+\eta_{1}\right) \exp \left(-1 / 2 u^{2}\left(1+\eta_{2}\right)\right)+\eta_{3} u^{2} \exp \left(3^{2}\right)
$$

where $\eta_{1}, \eta_{2}$ and $\eta_{3}$ depend on $n$ and $u$ but have absolute value less than $\epsilon$. Clearly we have $\lim _{n \rightarrow \infty} f_{n}(u)=\exp \left(-1 / 2 u^{2}\right)$ uniformly for $|u|<A$ as required.

3.2. We now produce an example to show that the Gaussian law fails for a set which is the union of two 2-dissociate sets. Let $G=Z(2)^{N}$, the set of all sequences $\mathrm{x}=\left\{x_{j}\right\}_{j=1}^{\infty}$ of elements of $\{0,1\}$ with componentwise addition modulo 2 as the group operation. For $F$ a nonempty finite subset of $N$, we define the character

$$
\chi(j: j \in F): \mathrm{x} \rightarrow \prod_{j \in F}(-1)^{x_{j}}
$$

For each $j \in N$, we set $X_{j}=\{\chi(1, j+1), \chi(j+1)\}$. We let $P=\{\chi(1, j+1)$, $\chi(j+1): j \in N\}$. Clearly we have $Q=P \cup P^{-1}=P$ and $Q_{k}=Q \cap X_{k}=X_{k}$. Further note that $Q$ is the union of the 2-dissociate sets $\{\chi(j+1): j \in N\}$ and $\{\chi(1, j+1): j \in N\}$. Let $u$ be the function which is identically equal to 1 on $Q$. We observe that, for $x \in Z(2)^{N}$,

$$
\begin{aligned}
S_{n}(\mathrm{x}) & =\sum_{j=1}^{n} \chi(1, j+1)(\mathrm{x})+\sum_{j=1}^{n} \chi(j+1)(\mathrm{x}) \\
& =\left(1+(-1)^{x_{1}}\right) \sum_{j=1}^{n} \chi(j+1)(\mathrm{x})
\end{aligned}
$$

and

$$
B_{n}=(2 n)^{1 / 2} \text {. }
$$


Now, for $y>0$, we have

$$
\begin{aligned}
\lambda\left\{S_{n}<y B_{n}\right\}=\lambda & \left(\left\{\mathrm{x}: x_{1}=1\right\} \cup\left\{\mathrm{x}: x_{1}=0 \text { and } 2 \sum_{j=1}^{n} \chi(1+j)(\mathrm{x})<y B_{n}\right\}\right) \\
& =1 / 2+1 / 2 \lambda\left\{2 \sum_{j=1}^{n} \chi(1+j)<y B_{n}\right\}
\end{aligned}
$$

while, for $y \leqslant 0$, we obtain

$$
\lambda\left\{S_{n}<y B_{n}\right\}=1 / 2 \lambda\left\{2 \sum_{j=1}^{n} \chi(1+j)<y B_{n}\right\} .
$$

Since $\{\chi(1+j): j \in N\}$ is a 2-dissociate set, we apply 3.1 to conclude that

$$
\lim _{n \rightarrow \infty} \lambda\left\{S_{n}<y B_{n}\right\}= \begin{cases}1 / 2+1 / 2 G_{1}\left(2^{-1 / 2} y\right) & \text { if } y>0, \\ 1 / 2 G_{1}\left(2^{-1 / 2} y\right) & \text { if } y \leqslant 0 .\end{cases}
$$

Note that the function on the right side of the above expression is discontinuous at zero.

3.3. A similar example shows that the Gaussian law fails to hold for 1-dissociate sets. Let $G=Z(4) \times Z(2)^{N}$. Each element $\mathrm{x}$ of $G$ can be expressed in the form $x=\left(x_{0}, x^{1}\right)$ where $x_{0} \in\{0,1,2,3\}$ and $x^{1} \in Z(2)^{N}$. Let $x_{j}$ be the character $x_{j}:\left(x_{0}, \mathbf{x}^{1}\right) \rightarrow i^{x}{ }^{0} \chi(j)\left(\mathbf{x}^{1}\right)$. Thus we readily calculate that

$$
\chi_{j}\left(x_{0}, \mathbf{x}^{1}\right)+\chi_{j}^{-1}\left(x_{0}, \mathbf{x}^{1}\right)= \begin{cases}2 \chi(j)\left(\mathbf{x}^{1}\right) & \text { if } x_{0}=0 \\ 0 & \text { if } x_{0}=1 \text { or } 3 \\ -2 \chi(j)\left(x^{1}\right) & \text { if } x_{0}=2\end{cases}
$$

The set $P=\left\{\chi_{j}: j \in N\right\}$ is easily shown to be a 1-dissociate set but it fails to be a 2 -Stečkin set. (Observe that $\chi_{j}^{2} \chi_{k}^{-2}=1$ for all $j, k \in N$.) A similar argument to the one given in 3.2 shows that the Gaussian law fails for the set $P$.

4

4.1. In this section, we suppose that $\Upsilon=\left\{X_{n}\right\}_{n=1}^{\infty}$ is a partition of a countably infinite subset of $\hat{G}$ by finite nonempty symmetric sets. Further we let $Q$ be an infinite symmetric subset of $\bigcup_{n=1}^{\infty} X_{n}$ and we let $u$ be a symmetric function defined on $Q$. We will obtain an estimate for

$$
\left\|\max _{r: m<r<m+p}\left(S_{r}-S_{m}\right)^{2}\right\|_{s} \text { for } m, p \in N, s>2,
$$

in the cases where $Q$ is a $\Lambda_{q}$ set for all $q>2$. This will enable us to show that

$$
\max _{r: p_{k}<r<p_{k+1}}\left|S_{r}\left(2 B_{r}^{2} \log \log B_{r}\right)^{-1 / 2}-S_{p_{k}}\left(2 B_{p_{k}}^{2} \log \log B_{p_{k}}\right)^{-1 / 2}\right|
$$


can be made small almost everywhere for a suitable sequence $\left\{p_{k}\right\}_{k=1}^{\infty}$ and large $k$. In establishing our result, we use properties of the dyadic representations of positive integers. We refine a technique used by Révész [1] in his proof of the law of the iterated logarithm for equinormed strongly multiplicative systems.

We will agree that all intervals used in this section are intervals of integers. For $k \in N$, we set

$$
E_{k}=\left\{\left\{\delta_{j}\right\}_{j=0}^{k-1}: \delta_{j}=0 \text { or } 1 \text { and } \delta_{0}=0\right\} \text {. }
$$

For $\left\{\delta_{j}\right\}_{j=0}^{n} \in E_{n+1}$, we use the symbol $\left(\delta_{0} \delta_{1} \cdots \delta_{n}\right)_{2}$ to denote the nonnegative integer $\Sigma_{j=0}^{n} \delta_{j} 2^{n-j}$ and refer to $\left(\delta_{0} \delta_{1} \cdots \delta_{n}\right)_{2}$ as the nth dyadic representation of $\Sigma_{j=0}^{n} \delta_{j} 2^{n-j}$. In what follows, we will agree to use the symbols $\delta_{j}$ and $\delta_{j}^{1}$ for each $j \in N$ only to denote elements of $\{0,1\}$. Further we will only have cause to refer to $n$th dyadic representations where $n$ is an unspecified positive integer. For $\Delta=\left\{\delta_{j}\right\}_{j=0}^{k-1} \in E_{k}$ where $0<k \leqslant n$, we put

$$
\Delta(0)=\left(\delta_{0} \delta_{1} \cdots \delta_{k-1} 00 \cdots 0\right)_{2} \text { and } \Delta(1)=\left(\delta_{0} \delta_{1} \cdots \delta_{k-1} 10 \cdots 0\right)_{2} \text {. }
$$

It is readily seen that each nonnegative integer $p<2^{n}$ has a unique $n$th dyadic representation and that $\left(\delta_{0} \delta_{1} \cdots \delta_{n}\right)_{2}<\left(\delta_{0}^{1} \delta_{1}^{1} \cdots \delta_{n}^{1}\right)_{2}$ if and only if $\delta_{r}<\delta_{r}^{1}$ where $r=\min \left\{k: \delta_{k} \neq \delta_{k}^{1}\right\}$. It will prove convenient to define for $p, n \in N$ and $k \in] 0, n], D(p, n, k)=\left\{\Delta \in E_{k}: \Delta(1) \leqslant p\right\}$.

4.2. The following lemma furnishes us with the properties of dyadic representations we need.

Lemma. Let $m \in Z$ and $p, n \in N$ such that $p<2^{n}$. For $k=1,2, \ldots, n$, define

$$
\Omega_{k}=\{[m+\Delta(0), m+\Delta(1)[: \Delta \in D(p, n, k)\}
$$

and set $\Omega=\bigcup_{k=1}^{n} \Omega_{k}$. Then the following are true:

(i) $\bigcup_{A \in \Omega} A=[m, m+p[$;

(ii) for each $x \in[m, m+p[$, the set $\{A \in \Omega: x \in A\}$ has at most $n$ elements;

(iii) if $f$ is a real-valued nondecreasing function defined on $[m, m+p]$, then

$$
\sum_{k=1}^{n} \sum_{\Delta \in D(p, n, k)} f(m+\Delta(1))-f(m+\Delta(0)) \leqslant n(f(m+p)-f(m)) .
$$

Proof. Without loss of generality, we may suppose that $m=0$. Let $p=$ $\left(\delta_{0} \delta_{1} \cdots \delta_{n}\right)_{2}$.

Let us prove (i). It is clear that $\bigcup_{A \in \Omega} A \subset[0, p$ [ since each set which is an element of $\Omega_{k}$ is a subset of [0,p [. To prove the reverse inclusion, let $x=$ $\left(\delta_{0}^{1} \delta_{1}^{1} \cdots \delta_{n}^{1}\right)_{2} \in\left[0, p\left[\right.\right.$. Then if $r=\min \left\{j: \delta_{j} \neq \delta_{j}^{1}\right\}$, we know that $\delta_{r}^{1}<\delta_{r}$ and therefore we have 


$$
x \in\left[\left(\delta_{0}^{1} \delta_{1}^{1} \cdots \delta_{r-1}^{1} 0 \cdots 0\right)_{2},\left(\delta_{0}^{1} \delta_{1}^{1} \cdots \delta_{r-1}^{1} 10 \cdots 0\right)_{2}[.\right.
$$

Further since $\delta_{k}^{1}=\delta_{k}$ for $k=0,1, \ldots, r-1$ and $\delta_{r}=1$, it is clear that the right endpoint of the above interval is at most equal to $p$ and the interval is consequently an element of $\Omega_{r}$. Part (i) is thus established.

To conclude that (ii) is true, we observe that the sets contained in $\Omega_{k}$ are mutually disjoint sets (that is, if $\Delta(0)<\Delta^{\prime}(0)$ for $\Delta, \Delta^{\prime} \in E_{k}$, then $\Delta(1)<$ $\left.\Delta^{\prime}(0)\right)$.

Finally we take up (iii). If $f$ is a real-valued nondecreasing function defined on $[0, p]$, then the function $g(x)=f(x+1)-f(x)$ defined on $[0, p[$ is a nonnegative function and we have

$$
f(b)-f(a)=\sum_{k \in[a, b]} g(k) \text { for } 0 \leqslant a<b \leqslant p .
$$

Thus we have

$$
\begin{aligned}
\sum_{k=1}^{n} \sum_{\Delta \in D(p, n, k)} f(\Delta(1))-f(\Delta(0))=\sum_{k=1}^{n} \sum_{\Delta \in D(p, n, k)} \sum_{x \in[\Delta(0), \Delta(1)]} g(x) \\
=\sum_{k=1}^{n} \sum_{A \in \Omega_{k}} \sum_{x \in A} g(x)=\sum_{A \in \Omega} \sum_{x \in A} g(x) \\
\quad \sum_{x \in \cup_{A \in \Omega} A} \text { (note that } \Omega \text { is the disjoint union of the } \Omega_{k} \text { 's) } \\
\quad \leqslant n \sum_{x \in[0, p[x \in A\} g(x)} g(x) \quad \text { (by parts (i) and (ii)) } \\
\leqslant n(f(p)-f(0)) . \quad \square
\end{aligned}
$$

4.3. For the remainder of this section, we will let $m$ denote an arbitrary nonnegative integer and $n$ an arbitrary positive integer. For $j \leqslant n$ and $\Delta \in E_{j}$, we define

$$
T(m, n, j, \Delta)=S_{m+\Delta(1)}-S_{m+\Delta(0)} .
$$

LEMMA. We have the following decomposition:

$$
S_{m}+\left(\delta_{0} \delta_{1} \cdots \delta_{n}\right)_{2}-S_{m}=\sum_{j=1}^{n} \delta_{j} T\left(m, n, j,\left\{\delta_{k}\right\}_{k=0}^{j-1}\right) .
$$

Proof. For $j \geqslant 1$ and $\delta_{j}=1$, we have $\delta_{j} T\left(m, n, j,\left\{\delta_{k}\right\}_{k=0}^{j-1}\right)=S_{m+\left(\delta_{0} \delta_{1} \cdots \delta_{j-1} \delta_{j} 0 \cdots 0\right)_{2}}-S_{m+\left(\delta_{0} \delta_{1} \cdots \delta_{j-1} 0 \cdots 0\right)_{2}}$. 
This inequality holds if $\delta_{j}=0$ since both sides are zero. By summing both sides and noting that all the middle terms in the right side cancel, we obtain

$$
\begin{aligned}
\sum_{j=1}^{n} \delta_{j} T\left(m, n, j,\left\{\delta_{k}\right\}_{k=0}^{j-1}\right) & =S_{m+\left(\delta_{0} \delta_{1} \cdots \delta_{n}\right)_{2}}-S_{m+\left(\delta_{0} 00 \cdots 0\right)_{2}} \\
& =S_{m+\left(\delta_{0} \delta_{1} \cdots \delta_{n}\right)_{2}}-S_{m} .
\end{aligned}
$$

4.4. Lemma. For $j \leqslant n$ and $\Delta \in E_{j}$, we have

$$
\int_{G} T^{2}(m, n, j, \Delta) d \lambda=B_{m+\Delta(1)}^{2}-B_{m+\Delta(0)}^{2} .
$$

Proof. Noting (4.1), we obtain

$$
\begin{array}{r}
\int_{G} T^{2}(m, n, j, \Delta) d \lambda=\sum_{k=\Delta(0)+1}^{\Delta(1)} \sum_{\chi \in Q_{m+k}} u(\chi) \int_{G} \chi T(m, n, j, \Delta) d \lambda \\
=\sum_{k=\Delta(0)+1}^{\Delta(1)} \sum_{x \in Q_{m+k}} u(\chi) \times \text { coefficient of } \chi^{-1} \text { in } T(m, n, j, \Delta) \\
=\sum_{k=\Delta(0)+1}^{\Delta(1)} \sum_{x \in Q_{m+k}}|u(x)|^{2}=B_{m+\Delta(1)}^{2}-B_{m+\Delta(0)}^{2} .
\end{array}
$$

4.5. We now suppose that $P$ is a $\Lambda_{s}$ set for all $s>2$ and let $Q=P \cup P^{-1}$. Note that $Q$ is also a $\Lambda_{s}$ set for all $s>2$ by (37.21)(a) of Hewitt and Ross, Vol. II [2]. Since a 1-Steckin set is a Sidon set (see Rider [1]), it will be a $\Lambda_{s}$ set for all $s>1$ by (37.10) of Hewitt and Ross, Vol. II. Hence our results will be valid whenever $P$ is a 1 -Steckin set.

THEOREM. For each $s>2$, there exists a constant $\phi_{s}>0$ dependent only on s such that

$$
\left\|T^{2}(m, n, j, \Delta)\right\|_{s} \leqslant \phi_{s}\left(B_{m+\Delta(1)}^{2}-B_{m+\Delta(0)}^{2}\right) .
$$

Proof. Since $T(m, n, j, \Delta)$ is a trignometric polynomial of elements of $Q$, there exists, by (37.7) of Hewitt and Ross, Vol. II, a constant $\phi_{s}>0$ such that

$$
\|T(m, n, j, \Delta)\|_{2 s}^{2} \leqslant \phi_{s}\|T(m, n, j, \Delta)\|_{2}^{2} .
$$

The result follows from 4.4 .

4.6. Lemma. Suppose that $p<2^{n}$. Then we have

$$
\max _{r: m<r<m+p}\left(S_{r}-S_{m}\right)^{2} \leqslant n\left(\sum_{k=1}^{n} \sum_{\Delta \in D(p, n, k)} T^{2}(m, n, k, \Delta)\right) .
$$


Proof. Let $r \in\left[m, n+p\left[\right.\right.$. Then we have $r=m+\left(\delta_{0} \delta_{1} \cdots \delta_{n}\right)_{2}$ where $\left(\delta_{0} \delta_{1} \cdots \delta_{n}\right)_{2}<p$ and we obtain

$$
\begin{aligned}
\left(S_{r}-S_{m}\right)^{2} & =\left(S_{\left.m+\left(\delta_{0} \delta_{1} \cdots \delta_{n}\right)_{2}-S_{m}\right)^{2}}\right. \\
& =\left(\sum_{k=1}^{n} \delta_{k} T\left(m, n, k,\left\{\delta_{j}\right\}_{j=1}^{k-1}\right)\right)^{2} \quad(\text { see } 4.3) \\
& \leqslant n\left(\sum_{k=1}^{n} \delta_{k} T^{2}\left(m, n, k,\left\{\delta_{j}\right\}_{j=1}^{k-1}\right)\right) \quad \text { (by Cauchy's inequality). }
\end{aligned}
$$

If $\delta_{k} T^{2}\left(m, n, k,\left\{\delta_{j}\right\}_{j=1}^{k-1}\right)$ appears as a nonzero term in our summation, we must have $\delta_{k}=1$ and hence $\left(\delta_{0} \delta_{1} \cdots \delta_{k-1} 10 \cdots 0\right)_{2} \leqslant\left(\delta_{0} \delta_{1} \cdots \delta_{n}\right)_{2}<p$ which implies that $\left\{\delta_{j}\right\}_{j=1}^{k-1} \in D(p, n, k)$. We conclude that

$$
\left(S_{r}-S_{m}\right)^{2} \leqslant n\left(\sum_{k=1}^{n} \sum_{\Delta \in D(p, n, k)} T^{2}(m, n, k, \Delta)\right) .
$$

Our result follows since $r$ was selected arbitrarily. $\quad$

4.7. We now piece together 4.5 and 4.6 to obtain the key theorem in this section.

TheOREM. Let $p \in N$ and $s>2$. Then setting $A_{s}=\phi_{s}(\log 2)^{-2}$ where $\phi_{s}$ is the constant appearing in 4.5 , we have

$$
\left\|\max _{r: m<r<m+p}\left(S_{r}-S_{m}\right)^{2}\right\|_{s} \leqslant A_{s}(\log (2 p))^{2}\left(B_{m+p}^{2}-B_{m}^{2}\right) .
$$

Proof. Choose $n \in N$ such that $2^{n-1} \leqslant p<2^{n}$. It is easily seen that

$$
n \log 2 \leqslant \log (2 p) \text {. }
$$

Using the inequality established in 4.6 , we obtain

$$
\begin{aligned}
\left\|\max _{r: m<r<m+p}\left(S_{r}-S_{m}\right)^{2}\right\|_{s} \leqslant n\left\|\sum_{k=1}^{n} \sum_{\Delta \in D(p, n, k)} T^{2}(m, n, k, \Delta)\right\|_{s} \\
\quad \leqslant \sum_{k=1}^{n} \sum_{\Delta \in D(p, n, k)}\left\|T^{2}(m, n, k, \Delta)\right\|_{s} \quad \text { (by Minkowski's inequality) } \\
\quad \leqslant n \phi_{s} \sum_{k=1}^{n} \sum_{\Delta \in D(p, n, k)}\left(B_{m+\Delta(1)}^{2}-B_{m+\Delta(0)}^{2}\right) \quad \text { (by 4.5) } \\
\leqslant n^{2} \phi_{s}\left(B_{m+p}^{2}-B_{m}^{2}\right) \quad \text { by (4.2(iii)) } \\
\leqslant A_{s}(\log 2 p)^{2}\left(B_{m+p}^{2}-B_{m}^{2}\right) \quad(\text { by 4.2)). }
\end{aligned}
$$


5.1. We now proceed to give the statement and proof of a law of the iterated logarithm for lacunary sets of characters. We recall the description of the problem given in 1.5. Before stating our key theorem, we need the following definition.

Definition. Let $\Upsilon, Q$ and $u$ be given. Suppose that $\left\{n_{k}\right\}_{k=1}^{\infty}$ is an increasing sequence of positive integers such that

$$
\log n_{k}\left(\log B_{n_{k}}\right)^{-\gamma}=O(1) \quad \text { as } k \rightarrow \infty \text { for some } \gamma>0 \text {. }
$$

Then if $\beta \geqslant 0$ is the greatest lower bound for all such $\gamma$, the sequence $\left\{n_{k}\right\}_{k=1}^{\infty}$ is called a $\beta$-sequence with respect to $\Upsilon, Q$ and $u$.

5.2. THEOREM. Let $P$ be a countably infinite subset of $G$ which is a $\Lambda_{q}$ set for all $q \geqslant 2$ and is $\Phi$-bounded by some positive number $\phi$ (see 2.13). Set $Q=P \cup P^{-1}$ and let $\Upsilon=\left\{X_{n}\right\}_{n=1}^{\infty}$ and $u$ be given such that $Q \subset \cup_{n=1}^{\infty} X_{n}$ and the following conditions are satisfied:

$$
\begin{aligned}
& \text { I. } B_{n} \rightarrow \infty \text { as } n \rightarrow \infty \text {, and } \\
& \text { II. } M_{n} B_{n}^{-1}\left(\log \log B_{n}\right)^{1 / 2}=o(1) \text { as } n \rightarrow \infty \text {. }
\end{aligned}
$$

Then if $\left\{n_{k}\right\}_{k=1}^{\infty}$ is a $\beta$-sequence, we have

$$
\limsup _{k \rightarrow \infty}\left|S_{n_{k}}\right|\left(2 B_{n_{k}}^{2} \log \log B_{n_{k}}\right)^{-1 / 2} \leqslant(2 \phi)^{1 / 2}(1+2 \beta)^{1 / 2} \quad \text { a.e. }
$$

Noting 2.14, we have

$\phi=1 / 2 m$ in (5.1) when $P$ is a 2 -Steckin set which is the union of $m$ sets having the property $\left(R_{2}\right)$, and

$\phi=m$ in (5.1) when $P$ is a 1-Steckin set which is the union of $m$ sets having the property $\left(R_{1}\right)$.

5.3. We accomplish the proof of 5.2 by a succession of lemmas. In each of these lemmas, we suppose that $\Upsilon, Q$ and $u$ are given and that $Q=P \cup P^{-1}$. In addition we suppose that $u$ satisfies conditions $I$ and II as stated in 5.2.

For notational convenience, we set

$$
T_{n}=\left(2 B_{n}^{2} \log \log B_{n}\right)^{1 / 2} \quad \text { for } n \in N .
$$

Lemma. Let $\gamma \in R ; \alpha, \beta>0$ and $r \in N$. Suppose that $P$ is $\Phi$-bounded by $\phi$ and let $\left\{p_{k}\right\}_{k=1}^{\infty}$ be a sequence of positive integers with the property that for each $j \in N$,

$$
B_{p_{k}} \geqslant \exp \left(\beta j^{\alpha}+\gamma\right) \text { if } k \geqslant r_{j} \text {. }
$$

It follows that

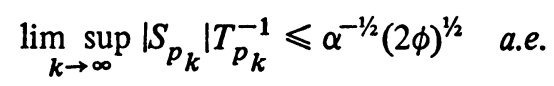


Proof. Choose $j_{0} \in N$ such that

$$
1 / 2 \beta j_{0}^{\alpha}+\gamma>e .
$$

For $k \geqslant r_{0}$, we have $B_{p_{k}} \geqslant \exp \left(\beta j_{0}^{\alpha}+\gamma\right)>\exp (e)$ and hence $\log \log B_{p_{k}}>1$. Suppose that we are given a positive number $\eta$ such that

$$
\eta<k_{1}(2 \phi)^{1 / 2} \alpha^{-1 / 2}
$$

(the constants $k_{1}, k_{2}$ and $k_{3}$ are those appearing in 2.13). For $k \geqslant j_{0}$, we put

$$
\Gamma_{k}=\lambda\left\{S_{p_{k}}>\left((2 \phi)^{1 / 2} \alpha^{-1 / 2}+\eta\right) T_{p_{k}}\right\}=\lambda\left\{S_{p_{k}} T_{p_{k}}^{-1}>(2 \phi)^{1 / 2} \alpha^{-1 / 2}+\eta\right\}
$$

For convenience, we define, for $k<r j_{0}, \Gamma_{k}=\lambda\left\{S_{p_{k}}>1\right\}$. It is well known that if $\Sigma_{k=1}^{\infty} \Gamma_{k}<\infty$ holds, then it will follow that

$$
\limsup _{k \rightarrow \infty} S_{p_{k}} T_{p_{k}}^{-1} \leqslant(2 \phi)^{1 / 2} \alpha^{-1 / 2}+\eta \text { a.e. }
$$

For all $n \geqslant p_{r j_{0}}$, let

$$
\theta_{n}=(2 \phi \alpha)^{-1 / 2} B_{n}^{-1}\left(2 \log \log B_{n}\right)^{1 / 2} .
$$

Then by condition II on $u$, there exists a positive integer $n^{\prime} \geqslant p_{\text {rij }}$ such that

$$
k_{2} \theta_{n} M_{n}<(2 \phi)^{-1 / 2} \alpha^{1 / 2} \eta \text { for } n \geqslant n^{\prime} \text {. }
$$

If $j_{1} \geqslant j_{0}$ is chosen such that $B_{n^{\prime}} \leqslant \exp \left(\beta j_{1}^{\alpha}+\gamma\right)$, then by (5.2) we know that if $k \geqslant r j_{1}$ holds, then $B_{p_{k}} \geqslant B_{n^{\prime}}$. Hence for $k \geqslant r_{1}$, we have $k_{2} \theta_{p_{k}} M_{p_{k}}<$ $(2 \phi)^{-1 / 2} \alpha^{1 / 2} \eta$. We can now apply 2.15 for each $k \geqslant r_{1}$ in the case where $W=$ $\bigcup_{j=1}^{p k_{1}} Q_{j}, \epsilon=(2 \phi)^{-1 / 2} \alpha^{1 / 2} \eta$ and $\theta=\theta_{p_{k}}$. Noting (5.4), we have $\epsilon<k_{1}$ and hence for all $y \in R$, we conclude that

$$
\lambda\left\{S_{p_{k}}>y\right\} \leqslant k_{3} \exp \left(\phi\left(1+\eta(2 \phi)^{-1 / 2} \alpha^{1 / 2}\right) \theta_{p_{k}}^{2} B_{p_{k}}^{2}-\theta_{p_{k}} y\right) .
$$

We set $y=\left((2 \phi)^{1 / 2} \alpha^{-1 / 2}+\eta\right) T_{p_{k}}$. Noting (5.5) and substituting for $\theta_{p_{k}}$ (see (5.6)) and $y$ in (5.7), we obtain after a straightforward calculation

$$
\Gamma_{k} \leqslant k_{3}\left(\log B_{p_{k}}\right)^{-\left(\alpha^{-1}+\eta(2 \phi)^{-1 / 2} \alpha^{-1 / 2}\right)} \text {. }
$$

It follows by (4.2) that, for $j \geqslant j_{1}$ and $0 \leqslant l<r$,

$$
\begin{aligned}
\Gamma_{r j+e} & \leqslant k_{3}\left(\log B_{p_{r j+e}}\right)^{-\left(\alpha^{-1}+\eta(2 \phi)^{-1 / 2} \alpha^{-1 / 2}\right)} \\
& \leqslant k_{3}\left(\beta j^{\alpha}+\gamma\right)^{-\left(\alpha^{-1}+\eta(2 \phi)^{-1 / 2} \alpha^{-1 / 2}\right)} .
\end{aligned}
$$

However, noting (5.3), we have for $j \geqslant j_{1} \geqslant j_{0}$

$$
\beta j^{\alpha}+\gamma \geqslant 1 / 2 \beta j^{\alpha}+1 / 2 \beta j_{0}^{\alpha}+\gamma>1 / 2 \beta j^{\alpha} \text {. }
$$

We conclude that for $j \geqslant j_{1}$ and $0 \leqslant l<r$ 


$$
\Gamma_{r j+e} \leqslant k_{3}(1 / 2 \beta)^{-\left(\alpha^{-1}+\eta(2 \phi)^{-1 / 2} \alpha^{-1 / 2}\right)_{j}-\left(1+\eta(2 \phi)^{-1 / 2} \alpha^{1 / 2}\right) .}
$$

It follows that $\Sigma_{k=1}^{\infty} \Gamma_{k}<\infty$ and hence we have

$$
\limsup _{k \rightarrow \infty} S_{p_{k}} T_{p_{k}}^{-1} \leqslant(2 \phi)^{1 / 2} \alpha^{-1 / 2}+\eta \text { a.e. }
$$

A similar argument using the function $-u$ instead of $u$ yields:

$$
\lim _{k \rightarrow \infty} \sup _{k}-S_{p_{k}} T_{p_{k}}^{-1} \leqslant(2 \phi)^{1 / 2} \alpha^{-1 / 2}+\eta \text { a.e. }
$$

Thus we conclude that

$$
\lim _{k \rightarrow \infty} \sup _{p_{k}} \mid T_{p_{k}}^{-1} \leqslant(2 \phi)^{1 / 2} \alpha^{-1 / 2}+\eta \quad \text { a.e. }
$$

Our lemma follows since $\eta$ can be made arbitrarily small.

5.4. Comment. If for each $n \in N$, we have $B_{n} \geqslant \exp \left(n^{n}\right)$, then we can apply 5.3 to conclude that $\lim \sup _{n \rightarrow \infty}\left|S_{n}\right| T_{n}^{-1}=0$ a.e. It is not obvious that this may occur if the rate of increase of the $B_{n}$ 's is restricted by condition II placed on $u$. However, if $u$ is identically equal to 1 on $Q$, we have $M_{n}=1$ and $B_{n}^{2}=\Sigma_{k=1}^{n}$ card $Q_{k}$. Condition II is clearly satisfied and by choosing the sequence $\Upsilon=\left\{X_{n}\right\}_{n=1}^{\infty}$ in such a way that card $Q_{n} \geqslant \exp \left(2 n^{n}\right)$ we find that $\lim \sup _{n \rightarrow \infty}\left|S_{n}\right| T_{n}^{-1}=0$ a.e.

If we specify that card $Q_{n}=O(1)$ as $n \rightarrow \infty$, then an argument which we outline below shows that $B_{n} \leqslant \exp \beta^{n}$ for some $\beta>0$ and hence 5.3 will not permit us to conclude that $\lim _{\sup _{n \rightarrow \infty}}\left|S_{n}\right| T_{n}^{-1} \leqslant 1$ a.e. If card $Q_{n} \leqslant B$ for all $n \in N$, then it follows readily from the definition that $M_{n} \geqslant B^{-1}\left(B_{n}^{2}-B_{n-1}^{2}\right)^{1 / 2}$. If $B_{n} B_{n-1}^{-1}$ is not bounded in $n$, it will follow that $M_{n} B_{n}^{-1}$ does not approach 0 thus contravening condition II. However, for $B_{n} B_{n-1}^{-1}=O(1)$ as $n \rightarrow \infty$, we readily conclude that $B_{n} \leqslant \exp \left(\beta^{n}\right)$ for some $\beta>0$.

5.5. We now choose a sequence $\left\{p_{k}\right\}_{k=1}^{\infty}$ satisfying (5.2) where $\alpha<1$ can be chosen in such a way that 4.7 can be used to show that

$$
\lim _{k \rightarrow \infty} \max _{n: p_{k}<n<p_{k+1}}\left|S_{n} T_{n}^{-1}-S_{p_{k}} T_{p_{k}}^{-1}\right|=0 \text { a.e. }
$$

The choice of $\alpha$ will depend on the rate of growth of $B_{n}$ with respect to $n$. The straightforward procedure would be to define $p_{k}$ by $p_{k}=\min \left\{n \in N: B_{n}^{2}>\right.$ $\exp \left(k^{\alpha}\right)$ \}. This is inadequate since in order to show (5.8) we require that $\left(B_{p_{k+1}}^{2}-B_{p_{k}}^{2}\right)\left(\exp (k+1)^{\alpha}-\exp \left(k^{\alpha}\right)^{-1}\right)$ be bounded for all $k$. In general this is not possible but for an appropriate choice of $\left\{p_{k}\right\}_{k=1}^{\infty}$ we can assure boundedness for all $k$ such that $p_{k+1}>p_{k}+1$ and this is sufficient.

Lemma. Let $\alpha \in[0,1]$ and $P$ be as in 5.3. Then there exists a strictly increasing sequence $\left\{p_{j}\right\}_{j=1}^{\infty}$ of positive integers with the following properties. We define the sequence $\left\{m_{j}\right\}_{j=1}^{\infty}$ of positive integers by setting 


$$
m_{j}=\max \left\{k \in N: B_{p_{j}}^{2}>\exp k\right\}
$$

Then we have:

(1) $m_{2 j} \geqslant j$ for each $j \in N$;

(2) $\lim \sup _{k \rightarrow \infty}\left|S_{p_{k}}\right| T_{p_{k}}^{-1} \leqslant(2 \phi)^{1 / 2} \alpha^{-1 / 2} \quad$ a.e.; and

(3) if $p_{k+1}>p_{k}+1$, then $B_{p_{k+1}}^{2}-B_{p_{k}}^{2}<2 \alpha m_{k}^{-1} \exp \left(m_{k}^{\alpha}\right)$.

PROOF. We note that $\left\{B_{n}^{2}\right\}_{n=1}^{\infty}$ is an unbounded nondecreasing sequence of nonnegative numbers. For each $k \in N$, we set

$$
A_{k}=\left\{j \in N: \exp k^{\alpha} \leqslant B_{j}^{2} \leqslant \exp (k+1)^{\alpha}\right\} .
$$

It is possible that $A_{k}$ is empty for some $k \in N$. We observe that if $j_{1} \in A_{k_{1}}$, $j_{2} \in A_{k_{2}}$ and $k_{1}<k_{2}$ then $j_{1}<j_{2}$. For each $k \in N$, we define

$$
q_{2 k-1}=\min \bigcup_{j=k}^{\infty} A_{j}, \quad \text { and } \quad q_{2 k}= \begin{cases}\max A_{k} & \text { if } A_{k} \neq \varnothing, \\ q_{2 k-1} & \text { if } A_{k}=\varnothing .\end{cases}
$$

It is clear that $\left\{q_{k}\right\}_{k=1}^{\infty}$ is a nondecreasing sequence with the property that

$$
q_{2 k+1} \leqslant q_{2 k}+1 \text { for all } k \in N \text {. }
$$

Choose $k_{0} \in N$ such that $k_{0}>2 \cdot 2(1-\alpha)^{-1}$. We let $\left\{p_{k}\right\}_{k=1}^{\infty}$ be the increasing sequence of distinct elements of $\left\{q_{k}\right\}_{k=k_{0}}^{\infty}$.

To show that (1) holds, we first observe that $p_{2 j} \geqslant q_{2 j}$ for all $j \in N$. Since $q_{2 j} \in A_{l}$ for some $l \geqslant j$, we conclude from (5.10) that $B_{p_{2 j}}^{2} \geqslant B_{q_{2 j}}^{2} \geqslant \exp (j)$. It follows that $m_{2 j} \geqslant j$ (see (5.9)).

In order to prove (2), it suffices to show that our sequence $\left\{p_{k}\right\}_{k=1}^{\infty}$ satisfies (5.2) for $\beta=1 / 2, \gamma=\theta$ and $r=2 j$. For $k \geqslant 2 j$, we have $p_{k} \geqslant p_{2 j}$ and hence

$$
\begin{aligned}
B_{p_{k}}^{2} & \geqslant B_{2 j}^{2}>\exp \left(m_{2 j}^{\alpha}\right) \quad \text { (note (5.9)) } \\
& \geqslant \exp \left(j^{\alpha}\right) \quad \text { (by part (1)). }
\end{aligned}
$$

The inequality $B_{p_{k}}>\exp \left(1 / 2 j^{\alpha}\right)$ holds for $k \geqslant 2 j$ and part (2) is thereby proved.

Finally we turn to (3). For all $k \in N$ we have $p_{k}=q_{h}$ and $p_{k+1}=q_{h+1}$ where $h=\max \left\{l \in N: p_{k}=q_{l}\right\}$. Noting (5.11), we infer that if $p_{k+1}>p_{k}+1$, then $q_{h+1}>q_{h}+1$ and hence $h$ is odd. Thus we conclude that for each $k \in N$ such that $p_{k+1}>p_{k}+1$, there exists $j \in N$ such that $p_{k}=q_{2 j-1}$ and $p_{k+1}=$ $q_{2 j}$. It follows that $A_{j} \neq \varnothing$ since $q_{2 j-1} \neq q_{2 j}$ and therefore

$$
\exp \left(j^{\alpha}\right) \leqslant B_{p_{k}}^{2} \leqslant B_{p_{k+1}}^{2}<\exp \left((j+1)^{\alpha}\right) .
$$

We have $B_{p_{k+1}}^{2}-B_{p_{k}}^{2}<\exp (j+1)^{\alpha}-\exp \left(j^{\alpha}\right)$. We have $j>1 / 2(2 j-1)>$ $2(1-\alpha)^{-1}$ by construction of $\left\{p_{k}\right\}_{k=1}^{\infty}$ and a simple argument which we omit yields:

$$
\exp (j+1)^{\alpha}-\exp \left(j^{\alpha}\right) \leqslant 2 j^{\alpha-1} \exp \left(j^{\alpha}\right) \quad \text { if } j>2^{(1-\alpha)^{-1}} .
$$


The proof of part (3) is complete since it is clear from (5.9) and (5.12) that $m_{k}=j$.

5.6. Before proving 5.2, we require a technical lemma.

LEMMA. Suppose that for a given sequence $\Upsilon=\left\{X_{n}\right\}_{n=1}^{\infty}$ of finite nonempty pairwise disjoint symmetric subsets of $\hat{G}$ and a strictly increasing sequence $\left\{n_{k}\right\}_{k=1}^{\infty}$ of positive integers, we define a new sequence $\Upsilon^{\dagger}=\left\{X_{k}^{\dagger}\right\}_{k=1}^{\infty}$ where

$$
X_{1}^{\dagger}=\bigcup_{j=1}^{n_{1}} X_{j} \text { and } X_{k}^{\dagger}=\bigcup_{j=n_{k-1}+1}^{n_{k}} X_{j} \text { for } k>1 \text {. }
$$

If corresponding to the terms $S_{n}, B_{n}$ and $M_{n}$ defined with respect to $\Upsilon$, we define $S_{k}^{\dagger}, B_{k}^{\dagger}$ and $M_{k}^{\dagger}$ with respect to $\Upsilon^{\dagger}$, then the following properties hold:

(1) $S_{k}^{\dagger}=S_{n_{k}}, B_{k}^{\dagger}=B_{n_{k}}$ and $M_{k}^{\dagger}=M_{n_{k}}$;

(2) if $u$ satisfies conditions I and II with respect to $\Upsilon$ (see 5.2) then $u$ satisfies conditions I and II with respect to $\Upsilon^{\dagger}$; and

(3) $\left\{n_{k}\right\}_{k=1}^{\infty}$ is a $\beta$-sequence with respect to $\Upsilon$ (see 5.1) if and only if $\{k\}_{k=1}^{\infty}$ is a $\beta$-sequence with respect to $\Upsilon^{\dagger}$.

ProOF. The proof is easy and is omitted.

5.7. PROOF of 5.2. In view of 5.6, we may suppose that $\left\{n_{k}\right\}_{k=1}^{\infty}$ is the sequence $\{n\}_{n=1}^{\infty}$. For $\epsilon>0$ and $\gamma>\beta$ given, we set $\alpha=(1+2 \gamma+\epsilon)^{-1}$. Then if $\left\{p_{k}\right\}_{k=1}^{\infty}$ is a sequence of positive integers having the properties (1), (2) and (3) of 5.5 for this choice of $\alpha$, we readily deduce from the second of these properties that if it is true that

$$
\lim _{k \rightarrow \infty} \max _{n: p_{k}<n<p_{k+1}}\left|S_{n} T_{n}^{-1}-S_{p_{k}} T_{p_{k}}^{-1}\right|=0 \text { a.e. }
$$

then it will follow that

$$
\lim _{n \rightarrow \infty} \sup _{n}\left|S_{n}\right| T_{n}^{-1} \leqslant(2 \phi)^{1 / 2}(1+2 \gamma+\epsilon)^{1 / 2} \quad \text { a.e. }
$$

Our proof will be complete since $\epsilon>0$ and $\gamma>\beta$ are arbitrarily chosen.

We begin by showing that

$$
\lim _{k \rightarrow \infty} \max _{n: p_{k}<n<p_{k+1}}\left|S_{n}-S_{p_{k}}\right| T_{p_{k}}^{-1}=0 \text { a.e. }
$$

We set

$$
s=\max \left\{2,2(\epsilon \alpha)^{-1}\right\} .
$$

Noting 5.5(1), we choose $k_{0} \in N$ such that for all $k \geqslant k_{0}$

$$
2 \alpha m_{k}^{\alpha-1}<1 \text {. }
$$

Now if $p_{k+1}=p_{k}+1$ for all $k \geqslant k_{0}$, then (5.13) is trivially true. Hence we suppose that $p_{k+1}>p_{k}+1$ holds for some $k \geqslant k_{0}$ and for any such $k$, we have by $5.5(3)$, 


$$
B_{p_{k+1}}^{2}-B_{p_{k}}^{2} \leqslant 2 \alpha m_{k}^{\alpha-1} \exp \left(m_{k}^{\alpha}\right) .
$$

By 4.7, we infer that

$$
\left\|\max _{n: p_{k}<n<p_{k+1}}\left(S_{n}-S_{p_{k}}\right)^{2}\right\|_{s} \leqslant A_{s}\left(\log \left(2\left(p_{k+1}-p_{k}\right)\right)\right)^{2}\left(B_{p_{k+1}}^{2}-B_{p_{k}}^{2}\right)
$$

where $A_{s}$ is the constant appearing in 4.7. We rewrite this inequality as

$$
\begin{aligned}
& \int_{G} \max _{n: p_{k}}<n<p_{k+1}\left(S_{n}-S_{p_{k}}\right)^{2 s} d \lambda \\
& \quad \leqslant A_{s}^{s}\left(\log \left(2\left(p_{k+1}-p_{k}\right)\right)\right)^{2 s}\left(B_{p_{k+1}}^{2}-B_{p_{k}}^{2}\right)^{s} .
\end{aligned}
$$

We estimate for $k \geqslant k_{0}$ :

$$
\begin{aligned}
\log \left(2\left(p_{k+1}-p_{k}\right)\right) & <\log \left(2 p_{k+1}\right) \leqslant 2 \log p_{k+1} \\
& <2 B\left(\log B_{p_{k+1}}\right)^{\alpha} \text { for some } B>0 \text { (see 5.1) } \\
& =2 \cdot 2^{-\alpha} B\left(\log B_{p_{k+1}}^{2}\right)^{\alpha} .
\end{aligned}
$$

Noting (5.17) and (5.9), we have for $k \geqslant k_{0}$ such that $p_{k+1}>p_{k}+1$.

$$
\begin{aligned}
\log \left(B_{p_{k+1}}^{2}\right) & \leqslant \log \left(B_{p_{k}}^{2}+2 \alpha m_{k}^{\alpha-1} \exp \left(m_{k}^{\alpha}\right)\right) \\
& \leqslant \log \left(\exp \left(\left(m_{k}+1\right)^{\alpha}\right)+2 m_{k}^{\alpha-1} \exp \left(m_{k}^{\alpha}\right)\right) \\
& <\log \left(2 \exp \left(\left(m_{k}+1\right)^{\alpha}\right)\right) \quad(\operatorname{see}(5.16)) \\
& <2\left(m_{k}+1\right)^{\alpha} \leqslant 2 \cdot 2^{\alpha} m_{k}^{\alpha} \\
& \leqslant A_{0} m_{k}^{2 \alpha \gamma s} m_{k}^{s(\alpha-1)}\left(\exp \left(m_{k}^{\alpha}\right)\right)^{s} .
\end{aligned}
$$

Substituting (5.19), (5.20) and (5.17) in (5.18), we have for $k \geqslant k_{0}$,

$$
\int_{G} \max _{n: p_{k}<n<p_{k+1}}\left(S_{n}-S_{p_{k}}\right)^{2 s} d \lambda \leqslant A_{0} m_{k}^{2 \alpha \gamma s} m_{k}^{s(\alpha-1)}\left(\exp \left(m_{k}^{\alpha}\right)\right)^{s}
$$

where $A_{0}$ is some constant independent of $k$. This inequality remains valid if $p_{k+1}=p_{k}+1$ since the left side is equal to 0 . We note that

$$
2 \alpha \gamma s+s(\alpha-1)=\alpha s(2 \gamma+1)-s=-\alpha s \epsilon \quad\left(\text { since } 2 \gamma+1=\alpha^{-1}-\epsilon\right)
$$

and that

$$
\begin{aligned}
T_{p_{k}}^{-2 s} & =2^{-s} B_{p_{k}}^{-2 s}\left(\log \log B_{p_{k}}\right)^{-s} \\
& \leqslant 2^{-s}\left(\exp \left(m_{k}^{\alpha}\right)\right)^{-s}\left(\log \log \exp \left(1 / 2 m_{k}^{\alpha}\right)\right)^{-s} \leqslant\left(\exp \left(m_{k}^{\alpha}\right)\right)^{-s} .
\end{aligned}
$$

Thus we obtain

$$
\int_{G: p_{k}<n<p_{k+1}}\left(S_{n}-S_{p_{k}}\right)^{2 s} T_{p_{k}}^{-2 s} d \lambda \leqslant A_{0} m_{k}^{-\alpha s} \leqslant A_{0} m_{k}^{-2} \quad \text { (see (5.15)). }
$$


Summing for $k \geqslant k_{0}$ and noting 5.5(2), we obtain

$$
\sum_{k=k_{0}}^{\infty} \int_{G} \max _{n: P_{k} \leqslant n<P_{k+1}}\left(S_{n}-S_{p_{k}}\right)^{2 s} T_{p_{k}}^{-2 s} d \lambda<\infty .
$$

We apply the monotone convergence theorem to obtain (5.14). Having established (5.14), we conclude the proof by showing (5.13) to be true. We let

$$
\begin{aligned}
H=\{t \in G: & \limsup _{k \rightarrow \infty}\left|S_{p_{k}}(t)\right| T_{p_{k}}^{-1} \leqslant(2 \phi)^{1 / 2} \alpha^{1 / 2} \text { and } \\
& \left.\lim _{k \rightarrow \infty} \max _{n: p_{k} \leqslant n<p_{k+1}}\left|S_{n}(t)-S_{p_{k}}(t)\right| T_{p_{k}}^{-1}=0\right\} .
\end{aligned}
$$

By 5.5(2) and (5.14), we conclude that $\lambda(H)=1$. Let $\eta>0$ and $t \in H$. Choose $k(t) \in N$ such that

(i) $B_{p_{k(t)}}>\exp (e)$,

(ii) $m_{k}^{\alpha-1}<(1 / 8)(2 \phi)^{-1 / 2} \alpha^{-1 / 2}$ for $k \geqslant k(t)$ (note that $m_{k} \rightarrow \infty$ by 5.5(1)),

(iii) $\max _{k>k(t)}\left|S_{p_{k}}(t)\right| T_{p_{k}}^{-1} \leqslant 2(2 \phi)^{1 / 2} \alpha^{-1 / 2}$, and

(iv) $\max _{n: p_{k} \leqslant n<p_{k+1}}\left|S_{n}(t)-S_{p_{k}}(t)\right| T_{p_{k}}^{-1} \leqslant \eta / 2$ for $k \geqslant k(t)$.

Our proof will be complete once we show for $k \geqslant k(t)$ that

$$
\max _{n: p_{k}<n<p_{k+1}}\left|S_{p_{k}}(t) T_{p_{k}}^{-1}-S_{n}(t) T_{n}^{-1}\right|<\eta / 2 .
$$

Clearly we may suppose that $p_{k+1}>p_{k}+1$. Let $n$ be any number such that $p_{k} \leqslant n<p_{k+1}$. Then since $T_{p_{k}} \leqslant T_{n}$, we have

$$
\begin{aligned}
& \left|S_{p_{k}}(t) T_{p_{k}}^{-1}-S_{n}(t) T_{n}^{-1}\right| \\
& \quad \leqslant\left|S_{p_{k}} T_{p_{k}}^{-1}-S_{p_{k}}(t) T_{n}^{-1}\right|+T_{n}^{-1}\left|S_{p_{k}}(t)-S_{n}(t)\right| \\
& \quad \leqslant\left|S_{p_{k}}(t)\right|\left(T_{p_{k}}^{-1}-T_{n}^{-1}\right)+T_{p_{k}}^{-1}\left|S_{p_{k}}(t)-S_{n}(t)\right| .
\end{aligned}
$$

A simple calculation yields for $B_{p_{k}} \geqslant \exp e$,

$$
T_{p_{k}}^{-1}-T_{n}^{-1} \leqslant T_{p_{k}}^{-1} B_{p_{k}}^{-2}\left(B_{n}^{2}-B_{p_{k}}^{2}\right) \leqslant T_{p_{k}}^{-1} B_{p_{k}}^{-2}\left(B_{p_{k+1}}^{2}-B_{p_{k}}^{2}\right) .
$$

Substituting this in (5.21), we obtain

$$
\begin{aligned}
& \left|S_{p_{k}}(t) T_{p_{k}}^{-1}-S_{n}(t) T_{n}^{-1}\right| \\
& \quad \leqslant\left|S_{p_{k}}(t)\right| T_{p_{k}}^{-1} B_{p_{k}}^{-2}\left(B_{p_{k+1}}^{2}-B_{p_{k}}^{2}\right)+T_{p_{k}}^{-1}\left|S_{p_{k}}(t)-S_{n}(t)\right| \\
& \quad \leqslant 2(2 \phi)^{1 / 2} \alpha^{-1 / 2} B_{p_{k}}^{-2}\left(B_{p_{k+1}}^{2}-B_{p_{k}}^{2}\right)+\eta / 2 \quad \text { (by (iii) and (iv)) } \\
& \quad<2(2 \phi)^{1 / 2} \alpha^{-1 / 2} 2 \alpha m_{k}^{\alpha-1}+\eta / 2 \quad \text { (by (5.9) and 5.5(3)) } \\
& \quad<\eta \quad \text { (by (ii)). }
\end{aligned}
$$


5.8. Remarks. (1) Our result, 5.2 , in the case where $k=n_{k}$ differs from that of the classical law of the iterated logarithm insofar as we impose an added condition on the $B_{k}$ 's. We note that

$$
\max _{\chi \in Q_{k}}|u(\chi)|^{2} \leqslant \sum_{\chi \in Q_{k}}|u(\chi)|^{2}=B_{k}^{2}-B_{k-1}^{2} .
$$

Thus we see that our function $u$ satisfies conditions I and II if

(i) $B_{n} \rightarrow \infty$, and

(ii) $\max _{k=1, \ldots, n} B_{n}^{-2}\left(B_{k}^{2}-B_{k-1}^{2}\right) \log \log B_{n}=o(1)$ as $n \rightarrow \infty$.

This observation enables us to deduce that in the case where $u$ satisfies conditions I and II, $\{n\}_{n=1}^{\infty}$ may be a $\beta$-sequence for a given $\beta \geqslant 0$ or may fail to be a $\beta$-sequence for any $\beta \geqslant 0$. Specifically we have:

(a) if $B_{n}=\exp (\log n)^{1 / \beta}$ for some $\beta>0$, then $u$ satisfies conditions I and II and $\{n\}_{n=1}^{\infty}$ is a $\beta$-sequence;

(b) if $B_{n}=\exp \left(\exp (\log \log n)^{2}\right)$ then $u$ satisfies conditions I and II and $\{n\}_{n=1}^{\infty}$ is a 0 -sequence;

(c) if $B_{n}=\log n$, then $u$ satisfies conditions I and II and $\{n\}_{n=1}^{\infty}$ fails to be a $\beta$-sequence for any $\beta>0$.

The verification of these claims is straightforward and we omit it.

(2) In the cases where $\beta=0$, the upper bounds given for the lim sup in 5.2 are the best possible for $P$, a 2-Steckin set and $P$, a 1 -Stečkin set. To see this, we examine the example given in 3.2. However, instead of setting $u$ identically equal to 1 , we define $u$ to be a function $u: \chi(1, j+1) \rightarrow u_{j}$ which satisfies conditions I and II and for which $\{n\}_{n=1}^{\infty}$ is a 0 -sequence. (For instance, set $u_{1}=$ $B_{1}$ and $u_{n}=\left(B_{n}^{2}-B_{n-1}^{2}\right)^{1 / 2}$ where $B_{n}=\exp \left(\exp (\log \log n)^{2}\right)$ as in (b) above.) We note that if $\mathrm{x} \in Z(2)^{N}$ is such that $x_{1}=0$, then

$$
S_{n}(\mathrm{x})=2 \sum_{j=1}^{n} u_{j} \chi(j+1)(\mathrm{x}) \text { and } B_{n}=2^{1 / 2}\left(\sum_{j=1}^{n} u_{j}^{2}\right)^{1 / 2} \text {. }
$$

It is easily verified that $\{\chi(j+1): j \in N\}$ is an independent set of functions on $Z(2)^{N}$ and we can apply 1.3 to conclude that for $x_{1}=0$,

$$
\begin{aligned}
\underset{n \rightarrow \infty}{\lim \sup _{n}} & S_{n}(\mathrm{x})\left(2 B_{n}^{2} \log \log B_{n}\right)^{-1 / 2} \\
= & 2^{1 / 2} \lim _{n \rightarrow \infty} \sum_{j=1}^{n} \chi(j+1)(\mathrm{x}) \\
& \times\left(2\left(\sum_{j=1}^{n} u_{j}^{2}\right) \log \log \left(2^{1 / 2}\left(\sum_{j=1}^{n} u_{j}^{2}\right)^{1 / 2}\right)\right)^{1 / 2} \\
= & 2^{1 / 2} \quad \text { for almost all } \mathrm{x} \text { such that } x_{1}=0 .
\end{aligned}
$$

A similar result is obtained for an arbitrary finite union of 2-dissociate sets viz. 
there exists a set $P$ which is the union of $m$ 2-dissociate sets such that for some $\Upsilon$ and $u$, we have

$$
\lim _{n \rightarrow \infty} S_{n}\left(2 B_{n}^{2} \log \log B_{n}\right)^{-1 / 2}=m^{1 / 2}
$$

on a set of positive measure. In this case, we examine the set $P=$ $\bigcup_{j=1}^{m}\left\{\chi\left(F_{j}, k+n\right): k \in N\right\}$ where $F_{1}, \ldots, F_{m}$ are distinct subsets of $\{1,2, \ldots, m\}$ and compute the lim sup on the set $\left\{x \in Z(2)^{N}: x_{1}=x_{2}=\cdots=x_{m}=0\right\}$.

A similar analysis using the example given in 3.3 yields that the upper bound given in 5.2 is attained for a set having the property $\left(R_{1}\right)$. Obviously we can extend this result to the finite union of sets having property $\left(R_{1}\right)$ in the manner suggested above.

(3) As remarked in 5.4, no positive lower bound for the lim sup exists unless we impose the restriction that card $X_{n}$ be bounded for all $n$. In any case, no such bound exists for the examples given in (2) since for these we have $S_{n}=$ 0 on a set of positive measure. In the case where card $X_{n}$ is bounded for all $n$ and $P$ is a set having the property $\left(R_{2}\right)$ (in particular a 2-dissociate set), we might expect a positive lower bound to exist in view of the fact that these sets are precisely the sets that satisfy the Gaussian law.

(4) For the special case where $G=T$, the circle group, our result 5.2 is only partially comparable with Mary Weiss's theorem (see 1.4). It is well known that the dual group $\hat{G}$, in this case, is isomorphic to the additive group $Z$. We observe that a 2-dissociate set $\left\{n_{k}: k \in N\right\}$ is one for which $\Sigma_{k \in F} \epsilon_{k} n_{k} \neq 0$ if $F$ is a nonempty finite subset of $N$ and $\epsilon_{k}= \pm 2, \pm 1$. It is readily shown that a Hadamard set $\left\{n_{k}: k \in N\right\}$ of positive integers for which $n_{k+1} / n_{k}>3$ for all $k \in N$ is a 2-dissociate set and any Hadamard set is the finite union of sets of this kind. However, a 2-dissociate set may fail to be the finite union of Hadamard sets (see Hewitt and Zuckerman [1]). Clearly our results apply to a larger class of lacunary sets than does Mary Weiss's theorem though our conclusions are weaker. It should also be noted that our results work for any partition $\Upsilon$ of $Z$ into finite symmetric sets whereas Mary Weiss's results are shown for the natural ordering of $N$.

\section{REFERENCES}

R. C. Baker

[1] On Wiener's theorem on Fourier Stielties coefficients and the Gaussian law, Proc. London Math. Soc. (3) 25 (1972), 525-542. MR 46 \#5929.

S. W. Drury

[1] The Fatou-Zygmund property for Sidon sets, Bull. Amer. Math. Soc. 80 (1974), 535-538.

Robert E. Edwards, Edwin Hewitt and Kenneth A. Ross

[1] Lacunarity for compact groups. I, Indiana Univ. Math. J. 21 (1971/72), 787-806. MR 45 \#6981.

[2] Lacunarity for compact groups. III, Studia Math. 44 (1972), 19-66. 
P. Erdös and I. S. Gál

[1] On the law of the iterated logarithm. I, II, Nederl. Akad. Wetensch. Proc. Ser. A 58 = Indag. Math. 17 (1955), 65-84. MR 16, 1016.

B. V. Gnedenko

[1] Course in the theory of probability, 4th ed., "Nauka", Moscow, 1965; English trans., Chelsea, New York, 1967. MR 36 \#13.

Edwin Hewitt and Kenneth A. Ross

[1] Abstract harmonic analysis. Vol. I: Structure of topological groups. Integration theory, group representations, Die Grundlehren der math. Wissenschaften, Band 115, Academic Press, New York; Springer-Verlag, Berlin, 1963. MR 28 \#158.

[2] Abstract harmonic analysis. Vol. II: Structure and analysis for compact groups. Analysis on locally compact Abelian groups, Die Grundlehren der math. Wissenschaften, Band 152, Academic Press, New York; Springer-Verlag, Berlin, 1970. MR 41 \#7378; erratum, 42, p. 1825.

Edwin Hewitt and Herbert S. Zuckerman

[1] Some theorems on lacunary Fourier series, with extensions to compact groups, Trans. Amer. Math. Soc. 93 (1959), 1-19. MR 21 \#7400.

A. Kolmogorov

[1] Über das Gesetz des iterierten Logarithmus, Math. Ann. 101 (1929), 126-135.

P. Révész

[1] Some remarks on strongly multiplicative systems, Acta Math. Acad. Sci. Hungar. 16 (1965), 441-446. MR 32 \#3097.

Daniel G. Rider

[1] Gap series on groups and spheres, Canad. J. Math. 18 (1966), 389-398. MR 32 \#8047.

R. Salem and A. Zygmund

[1] On lacunary trigonometric series. I, Proc. Nat. Acad. Sci. U.S.A. 33 (1947), 333338. MR 9, 181.

[2] On lacunary trigonometric series. II, Proc. Nat. Acad. Sci. U.S.A. 34 (1948), 5462. MR 9, 425.

[3] La loi du logarithme itéré pour les séries trigonométriques lacunaires, Bull. Sci.

S. B. Stečkin Math. (2) 74 (1950), 209-224. MR 12, 605.

[1] On absolute convergence of Fourier series, Izv. Akad. Nauk SSSR Ser. Mat. 20 (1956), 385-412. (Russian) MR 18, 126.

Mary Weiss

[1] The law of the iterated logarithm for lacunary trigonometric series, Trans. Amer. Math. Soc. 91 (1959), 444-469. MR 21 \#396.

DEPARTMENT OF MATHEMATICS, UNIVERSITY OF MELBOURNE, PARKVILLE, VICTORIA 3052, AUSTRALIA 